\title{
A THOM-SEBASTIANI THEOREM IN CHARACTERISTIC $p$
}

\author{
LEI FU
}

\begin{abstract}
Let $k$ be a perfect field of characteristic $p, X_{i}(i=1,2)$ smooth $k$-schemes, $f_{i}: X_{i} \rightarrow \mathbb{A}_{k}^{1}$ two $k$-morphisms of finite type, and $f: X_{1} \times_{k} X_{2} \rightarrow \mathbb{A}_{k}^{1}$ the morphism defined by $f\left(z_{1}, z_{2}\right)=f_{1}\left(z_{1}\right)+f_{2}\left(z_{2}\right)$. For each $i \in\{1,2\}$, let $x_{i}$ be a $k$-rational point in the fiber $f_{i}^{-1}(0)$ such that $f_{i}$ is smooth on $X_{i}-\left\{x_{i}\right\}$. Using the $\ell$-adic Fourier transformation and the stationary phase principle of Laumon, we prove that the vanishing cycles complex of $f$ at $x=\left(x_{1}, x_{2}\right)$ is the convolution product of the vanishing cycles complexes of $f_{i}$ at $x_{i}(i=1,2)$.
\end{abstract}

\section{Introduction}

Let $f_{i}:\left(\mathbb{C}^{n_{i}}, 0\right) \rightarrow(\mathbb{C}, 0)(i=1,2)$ be two germs of analytic functions with isolated critical points, and consider the germ $f:\left(\mathbb{C}^{n_{1}+n_{2}}, 0\right) \rightarrow(\mathbb{C}, 0)$ defined by $f\left(z_{1}, z_{2}\right)=$ $f_{1}\left(z_{1}\right)+f_{2}\left(z_{2}\right)$. The classical Thom-Sebastiani theorem $([15])$ states that the vanishing cycles complex of $f$ is isomorphic to the tensor product of those of $f_{1}$ and $f_{2}$. This theorem is not correct for a general field $k$. Indeed, let $f: \mathbb{A}_{k}^{n} \rightarrow \mathbb{A}_{k}^{1}$ be the $k$-morphism defined by the quadratic polynomial

$$
f=z_{1}^{2}+\cdots+z_{n}^{2}
$$

for a field $k$ of characteristic $\neq 2, \ell$ a prime number distinct from the characteristic of $k, \overline{\mathbb{Q}}_{\ell}$ an algebraic closure of the field $\mathbb{Q}_{\ell}$ of $\ell$-adic numbers, and $R \Phi_{f}\left(\overline{\mathbb{Q}}_{\ell}\right)$ the vanishing cycles complex for the constant $\ell$-adic sheaf $\overline{\mathbb{Q}}_{\ell}$ relative to the morphism $f$. By $[5, \mathrm{XV}$ $2.2]$, we have $R^{i} \Phi_{f}\left(\overline{\mathbb{Q}}_{\ell}\right)=0$ for $i \neq n-1$, and $R^{n-1} \Phi_{f}\left(\overline{\mathbb{Q}}_{\ell}\right)$ is supported at the origin. Let $K=k((t))$ be the formal Laurent series field. The stalk $\left(R^{n-1} \Phi_{f}\left(\overline{\mathbb{Q}}_{\ell}\right)\right)_{0}$ at the origin is a one-dimensional $\overline{\mathbb{Q}}_{\ell}$-vector space on which $\operatorname{Gal}(\bar{K} / K)$ acts continuously. By $[5, \mathrm{XV} 2.2 .5 \mathrm{D}, \mathrm{E}]$, we have

$$
\left(R^{n-1} \Phi_{f}\left(\overline{\mathbb{Q}}_{\ell}\right)\right)_{0} \cong V_{\chi}\left(-\left[\frac{n}{2}\right]\right)
$$

for some one-dimensional $\overline{\mathbb{Q}}_{\ell}$-vector space $V_{\chi}$ on which $\operatorname{Gal}(\bar{K} / K)$ acts through a character

$$
\chi: \operatorname{Gal}(\bar{K} / K) \rightarrow \overline{\mathbb{Q}}_{\ell}^{*}
$$

of order 2 , where $\left[\frac{n}{2}\right]$ denotes the largest integer that is less than or equal to $\frac{n}{2}$. Since $\left[\frac{n}{2}\right]$ in the Tate twist is not linear in $n$, one sees immediately that the Thom-Sebastiani

Received by the editors November 7, 2012.

1991 Mathematics Subject Classification. 14F20.

Key words and phrases. vanishing cycles, nearby cycles, local Fourier transformation, perverse sheaf. 
theorem in terms of tensor product does not hold for a general field $k$ and quadratic

$$
f_{1}=z_{1}^{2}+\cdots+z_{n_{1}}^{2}, \quad f_{2}=z_{n_{1}+1}^{2}+\cdots+z_{n_{1}+n_{2}}^{2}
$$

if both $n_{1}$ and $n_{2}$ are odd.

In [1] (unpublished), Deligne studies the analog of the Thom-Sebastiani theorem for the variation morphism, and realizes that the tensor product in the Thom-Sebastiani theorem should be replaced by the convolution product. Deligne constructs the vanishing cycles complex $V_{f}$ and the monodromy $T_{f}$ associated to $f$ with the aid of those $\left(V_{f_{i}}, T_{f_{i}}\right)(i=1,2)$ associated to $f_{i}$. Topologically, $V_{f}$ retracts to the join $V_{f_{1}} * V_{f_{2}}$. This should be translated using convolution.

In [16], using the Fourier transformation, the formula

$$
\int e^{i t f}=\int e^{i t f_{1}} \int e^{i t f_{2}}
$$

and the asymptotic expansions of such integrals for $t \rightarrow \infty$, Varchenko proves a Thom-Sebastiani theorem for the Hodge spectrum. Inspired by Varchenko's method, we prove a Thom-Sebastiani theorem in characteristic $p$ using the Deligne-Fourier transformation.

The Thom-Sebastiani theorem has been studied extensively over $\mathbb{C}$. See, for example, $[6,7,13,14]$. Due to the use of the Deligne-Fourier transformation, our method cannot be directly applied to the characteristic 0 case. But it suggests that one can use the Fourier transformation for $D$-modules to study the Thom-Sebastiani theorem over $\mathbb{C}$.

\section{Main result}

Throughout this paper, $k$ is a perfect field of characteristic $p$ and $\ell$ is a prime number distinct from $p$. We assume that for any finite extension $k^{\prime}$ of $k$, the groups $H^{i}\left(\operatorname{Gal}\left(k^{\prime} / k\right), \mathbb{Z} / \ell \mathbb{Z}\right)(i \in \mathbb{N})$ are finite. For any scheme $X$ of finite type over $k$, the category $D_{c}^{b}\left(X, \overline{\mathbb{Q}}_{\ell}\right)$ constructed in $[4,1.1]$ is a triangulated category. Let $S$ be a Henselian trait of equal characteristic $p$ with generic point $\eta$ and special point $s$ such that $k(s)=k$, let $f: X \rightarrow S$ be a morphism of finite type, and let $K$ be an object in $D_{c}^{b}\left(X, \overline{\mathbb{Q}}_{\ell}\right)$. We refer the reader to $[5, \mathrm{XIII}]$ for the definitions and properties of the nearby cycles complex $R \Psi_{f}(K)$ and the vanishing cycles complex $R \Phi_{f}(K)$ relative to the morphism $f$. We also denote $R \Psi_{f}$ and $R \Phi_{f}$ by $R \Psi$ and $R \Phi$ for convenience.

Lemma 2.1. Let $X \rightarrow S$ be a morphism of finite type, let $x$ be a $k$-rational point in the special fiber $X_{s}$, and let $K \in$ ob $D_{c}^{b}\left(X, \overline{\mathbb{Q}}_{\ell}\right)$. Suppose $X-\{x\} \rightarrow S$ is smooth and the sheaves $\left.\mathscr{H}^{q}(K)\right|_{X-\{x\}}$ are lisse for all $q$ :

(i) $\left.R \Phi(K)\right|_{X_{\bar{s}}-\{x\}}=0$.

(ii) Suppose furthermore that $X$ is pure of dimension $n$ and regular at $x$, and $K$ is a lisse sheaf. Then $R^{i} \Phi(K)$ vanishes for $i \neq n-1$, and $R^{n-1} \Phi(K)$ is a skyscraper sheaf on $X_{\bar{s}}$ supported at $x$.

Proof. See [10, 2.10]. We give a proof here for completeness. (i) follows from the smooth base change theorem. Under the assumption of (ii), $K[n]$ is a perverse sheaf on $X$. By $[9,4.6], R \Phi(K[n])[-1]$ is perverse. Combined with (i), we see that $R \Phi(K[n])[-1]$ is a perverse sheaf on $X_{\bar{s}}$ supported at $x$. Our assertion follows. 
Let $\mathbb{A}_{(0)}^{1}$ be the Henselization of $\mathbb{A}_{k}^{1}$ at 0 , let $\eta_{0}$ be its generic point, and let $j$ : $\eta_{0} \hookrightarrow \mathbb{A}_{(0)}^{1}$ be the canonical open immersion. We can identify a $\operatorname{Gal}\left(\bar{\eta}_{0} / \eta_{0}\right)$-module with a sheaf on $\eta_{0}$. Let $\left(\mathbb{A}_{k}^{1} \times_{k} \mathbb{A}_{k}^{1}\right)_{(0,0)}$ be the Henselization of $\mathbb{A}_{k}^{1} \times_{k} \mathbb{A}_{k}^{1}$ at $(0,0)$, and let

$$
\tilde{p}_{1}, \tilde{p}_{2}, \tilde{a}:\left(\mathbb{A}_{k}^{1} \times_{k} \mathbb{A}_{k}^{1}\right)_{(0,0)} \rightarrow \mathbb{A}_{(0)}^{1}
$$

be the morphisms induced by the two projections $p_{1}, p_{2}: \mathbb{A}_{k}^{1} \times_{k} \mathbb{A}_{k}^{1} \rightarrow \mathbb{A}_{k}^{1}$ and the addition $a: \mathbb{A}_{k}^{1} \times_{k} \mathbb{A}_{k}^{1} \rightarrow \mathbb{A}_{k}^{1}$ of the algebraic group $\mathbb{A}_{k}^{1}$, respectively. Let $V_{1}$ and $V_{2}$ be $\overline{\mathbb{Q}}_{\ell}$-representations of $\operatorname{Gal}\left(\bar{\eta}_{0} / \eta_{0}\right)$, and regard them as sheaves on $\eta_{0}$. By $[12,2.7 .1 .3]$, the vanishing cycles complex $R \Phi_{\tilde{a}}\left(\tilde{p}_{1}^{*} j_{!} V_{1} \otimes^{L} \tilde{p}_{2}^{*} j_{!} V_{2}\right)$ relative to the morphism $\tilde{a}$ is nonzero only at $(0,0)$ and at degree 1 . Recall that the convolution product of $V_{1}$ and $V_{2}([12,2.7 .2])$ is the $\operatorname{Gal}\left(\bar{\eta}_{0} / \eta_{0}\right)$-module

$$
V_{1} * V_{2}=R^{1} \Phi_{\tilde{a}}\left(\tilde{p}_{1}^{*} j_{!} V_{1} \otimes \tilde{p}_{2}^{*} j_{!} V_{2}\right)_{(0,0)}
$$

If $\mathscr{V}_{1}$ and $\mathscr{V}_{2}$ are objects in $D_{c}^{b}\left(\eta_{0}, \overline{\mathbb{Q}}_{\ell}\right)$, we define their convolution product to be

$$
\mathscr{V}_{1} \mathscr{V}_{2}=R \Phi_{\tilde{a}}\left(\tilde{p}_{1}^{*} j_{!} \mathscr{V}_{1} \otimes^{L} \tilde{p}_{2}^{*} j ! \mathscr{V}_{2}\right)_{(0,0)}
$$

If $\mathscr{V}_{i}(i=1,2)$ are objects $D_{c}^{b}\left(\eta_{0}, \overline{\mathbb{Q}}_{\ell}\right)$ defined by the $\operatorname{Gal}\left(\bar{\eta}_{0} / \eta_{0}\right)$-modules $V_{i}(i=1,2)$, respectively, then $\left(\mathscr{V}_{1} \underline{*}_{2}\right)[1]$ is the object in $D_{c}^{b}\left(\eta_{0}, \overline{\mathbb{Q}}_{\ell}\right)$ defined by the $\operatorname{Gal}\left(\bar{\eta}_{0} / \eta_{0}\right)$ module $V_{1} * V_{2}$.

Our main result is the following.

Theorem 2.2. Let $f_{i}: X_{i} \rightarrow \mathbb{A}_{k}^{1}(i=1,2)$ be two flat $k$-morphisms of finite type, $K_{i} \in$ ob $D_{c}^{b}\left(X_{i}, \overline{\mathbb{Q}}_{\ell}\right), X=X_{1} \times_{S} X_{2}, K=K_{1} \otimes^{L} K_{2}$, and $f: X=X_{1} \times_{k} X_{2} \rightarrow \mathbb{A}_{k}^{1}$ the morphism defined by

$$
f\left(z_{1}, z_{2}\right)=f_{1}\left(z_{1}\right)+f_{2}\left(z_{2}\right)
$$

For each $i \in\{1,2\}$, let $x_{i}$ be a k-rational point in the fiber $f_{i}^{-1}(0)$. Suppose that $X_{i}$ is regular, $\mathscr{H}^{q}\left(K_{i}\right)$ are lisse for all $q$, and $\left.f_{i}\right|_{X_{i}-\left\{x_{i}\right\}}$ is smooth. Denoted by $x$ the $k$-rational point $\left(x_{1}, x_{2}\right)$ on $X$. Denoted, respectively, by $R \Phi_{f}$ and $R \Phi_{f_{i}}$ the vanishing cycles functors relative to the morphisms

$$
X \times_{\mathbb{A}_{k}^{1}} \mathbb{A}_{(0)}^{1} \rightarrow \mathbb{A}_{(0)}^{1}, \quad X_{i} \times_{\mathbb{A}_{k}^{1}} \mathbb{A}_{(0)}^{1} \rightarrow \mathbb{A}_{(0)}^{1}
$$

obtained from $f$ and $f_{i}$ by base change.

(i) $X$ is regular, and $\left.f\right|_{X-\{x\}}$ is smooth.

(ii) As objects in $D_{c}^{b}\left(\eta_{0}, \overline{\mathbb{Q}}_{\ell}\right)$, we have a canonical isomorphism

$$
\left(R \Phi_{f_{1}}\left(K_{1}\right)\right)_{x_{1} *}\left(R \Phi_{f_{2}}\left(K_{2}\right)\right)_{x_{2}} \cong\left(R \Phi_{f}(K)\right)_{x} .
$$

(iii) Suppose furthermore that for each $i, K_{i}$ is a lisse sheaf. Let $n_{i}=\operatorname{dim} \mathscr{O}_{X_{i}, x_{i}}$, and let $n=n_{1}+n_{2}$. Then as $\operatorname{Gal}\left(\bar{\eta}_{0} / \eta_{0}\right)$-modules, we have a canonical isomorphism

$$
\left(R^{n_{1}-1} \Phi_{f_{1}}\left(K_{1}\right)\right)_{x_{1}} *\left(R^{n_{2}-1} \Phi_{f_{2}}\left(K_{2}\right)\right)_{x_{2}} \cong\left(R^{n-1} \Phi_{f}(K)\right)_{x}
$$

\section{Example 2.3.}

By our construction, the canonical isomorphisms in Theorem 1.2 (ii)-(iii) are associative. Let us use Theorem 2.2 to calculate the vanishing cycles complex for the 
quadratic morphism

$$
f: \mathbb{A}_{k}^{n} \rightarrow \mathbb{A}_{k}^{1}, \quad f(z)=z_{1}^{2}+\cdots+z_{n}^{2}
$$

in the case where char $k \neq 2$.

First consider the case where $n=1$. Then $f$ is a finite morphism. By the proper base change theorem, we have

$$
R \Phi_{f}\left(\overline{\mathbb{Q}}_{\ell}\right) \cong R \Phi\left(f_{*} \overline{\mathbb{Q}}_{\ell}\right)
$$

We have

$$
f_{*} \overline{\mathbb{Q}}_{\ell} \cong \overline{\mathbb{Q}}_{\ell} \bigoplus j_{!} \mathscr{K}_{\chi_{2}}
$$

where $j: \mathbb{A}_{k}^{1}-\{0\} \hookrightarrow \mathbb{A}_{k}^{1}$ is the canonical open immersion and $\mathscr{K}_{\chi_{2}}$ is the Kummer sheaf on $\mathbb{A}_{k}^{1}-\{0\}$ associated to the nontrivial character

$$
\chi_{2}: \boldsymbol{\mu}_{2}(k) \rightarrow\{ \pm 1\}
$$

of order 2. Let $K=k((t))$ be the formal Laurent series field, and let $V_{\chi_{2}}$ be the one-dimensional $\overline{\mathbb{Q}}_{\ell}$-vector space on which $\operatorname{Gal}(\bar{K} / K)$ acts through the character

$$
\operatorname{Gal}(\bar{K} / K) \rightarrow \overline{\mathbb{Q}}_{\ell}^{*}, \quad \sigma \mapsto \chi_{2}\left(\frac{\sigma(\sqrt{t})}{\sqrt{t}}\right) .
$$

For the vanishing cycles functor with respect to the identity morphism on $\mathbb{A}_{(0)}^{1}$, we have

$$
R \Phi\left(\overline{\mathbb{Q}}_{\ell}\right)=0, \quad R \Phi\left(j_{!} \mathscr{K}_{\chi_{2}}\right)=V_{\chi_{2}}
$$

Indeed, let $\eta$ (resp. 0) be the generic (resp. closed) point of $\mathbb{A}_{(0)}^{1}$. For any object $K$ in $D_{c}^{b}\left(\mathbb{A}_{(0)}^{1}, \overline{\mathbb{Q}}_{\ell}\right), R \Phi(K)$ can be identified with the cone of the specialization morphism $K_{\overline{0}} \rightarrow K_{\bar{\eta}}$. If $K=\overline{\mathbb{Q}}_{\ell}$, the specialization morphism is an isomorphism and hence, $R \Phi\left(\overline{\mathbb{Q}}_{\ell}\right)=0$. If $K=j_{!} \mathscr{K}_{\chi_{2}}$, we have $K_{\overline{0}}=0$ and $K_{\bar{\eta}} \cong V_{\chi_{2}}$. So, we have $R \Phi\left(j_{!} \mathscr{K}_{\chi_{2}}\right)=$ $V_{\chi_{2}}$. We thus have

$$
R^{0} \Phi_{f}\left(\overline{\mathbb{Q}}_{\ell}\right) \cong V_{\chi_{2}}
$$

for the quadratic morphism $f(z)=z^{2}$. By Theorem 1.2, for $f=z_{1}^{2}+\cdots+z_{n}^{2}$, we have

$$
\left(R \Phi_{f}^{n-1}\left(\overline{\mathbb{Q}}_{\ell}\right)\right)_{0} \cong *^{n}\left(V_{\chi_{2}}\right)
$$

where $*^{n}$ denotes the $n$th convolution power. Let us calculate this convolution power.

Fix a nontrivial additive character $\psi: \mathbb{Z} / p \rightarrow \overline{\mathbb{Q}}_{\ell}^{*}$. Let

$$
g\left(\chi_{2}, \psi\right)=\sum_{x \in \mathbb{F}_{p}^{*}}\left(\frac{x}{p}\right) \psi(x)
$$

be the quadratic Gauss sum, and let $G\left(\chi_{2}, \psi\right)$ is be the one-dimensional $\overline{\mathbb{Q}}_{\ell^{-}}$vector space with $\operatorname{Gal}\left(\overline{\mathbb{F}}_{p} / \mathbb{F}_{p}\right)$ action, so that the geometric Frobenius element acts by scalar multiplication by $-g\left(\chi_{2}, \psi\right)$. Let $V_{\epsilon^{m}}$ be the one-dimensional $\overline{\mathbb{Q}}_{\ell^{-v}}$ ector space with $\operatorname{Gal}\left(\overline{\mathbb{F}}_{p} / \mathbb{F}_{p}\right)$ action, so that the geometric Frobenius element acts by scalar multipli-

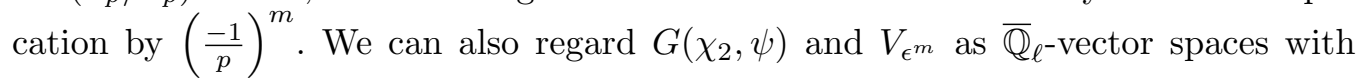
$\operatorname{Gal}(\bar{K} / K)$ action and with $\operatorname{Gal}(\bar{k} / k)$ action through the canonical homomorphisms

$$
\operatorname{Gal}(\bar{K} / K) \rightarrow \operatorname{Gal}(\bar{k} / k) \rightarrow \operatorname{Gal}\left(\overline{\mathbb{F}}_{p} / \mathbb{F}_{p}\right)
$$


We have

$$
G\left(\chi_{2}, \psi\right) \otimes G\left(\chi_{2}, \psi\right) \cong V_{\epsilon}(-1) .
$$

This follows from the formula

$$
\left(g\left(\chi_{2}, \psi\right)\right)^{2}=\left(\frac{-1}{p}\right) p
$$

for the quadratic Gauss sum. We thus have the following formula for the $n$th tensor power

$$
\otimes^{n}\left(V_{\chi_{2}} \otimes G\left(\chi_{2}, \psi\right)\right) \cong \begin{cases}V_{\epsilon^{m}}(-m) & \text { if } n=2 m \text { is even, } \\ V_{\chi_{2}} \otimes G\left(\chi_{2}, \psi\right) \otimes V_{\epsilon^{m}}(-m) & \text { if } n=2 m+1 \text { is odd }\end{cases}
$$

Let $\mathscr{F}^{\left(0, \infty^{\prime}\right)}$ be Laumon's local Fourier transformation [12, 2.4.2.3] associated with the character $\psi$. By $[12,2.5 .3 .1]$, we have

$$
\mathscr{F}^{\left(0, \infty^{\prime}\right)}\left(V_{\chi_{2}}\right) \cong V_{\chi_{2}} \otimes G\left(\chi_{2}, \psi\right) .
$$

By $[12,2.7 .2 .2(\mathrm{i})]$, we have

$$
\begin{aligned}
& \mathscr{F}^{\left(0, \infty^{\prime}\right)}\left(*^{n}\left(V_{\chi_{2}}\right)\right) \cong \otimes^{n} \mathscr{F}^{\left(0, \infty^{\prime}\right)}\left(V_{\chi_{2}}\right), \\
& \cong \otimes^{n}\left(V_{\chi_{2}} \otimes G\left(\chi_{2}, \psi\right)\right) \text {, } \\
& \cong \begin{cases}V_{\epsilon^{m}}(-m) & \text { if } n=2 m \text { is even, } \\
V_{\chi_{2}} \otimes G\left(\chi_{2}, \psi\right) \otimes V_{\epsilon^{m}}(-m) & \text { if } n=2 m+1 \text { is odd. }\end{cases}
\end{aligned}
$$

On the other hand, the calculation of $[12,2.5 .3 .1]$ shows that

$$
\begin{aligned}
\mathscr{F}^{\left(0, \infty^{\prime}\right)}\left(V_{\epsilon^{m}}(-m)\right) & \cong V_{\epsilon^{m}}(-m), \\
\mathscr{F}^{\left(0, \infty^{\prime}\right)}\left(V_{\chi_{2}} \otimes V_{\epsilon^{m}}(-m)\right) & \cong V_{\chi_{2}} \otimes G\left(\chi_{2}, \psi\right) \otimes V_{\epsilon^{m}}(-m) .
\end{aligned}
$$

By the inversion formula for the local Fourier transformation [12, 2.4.3 (i)c)], we must have

$$
*^{n}\left(V_{\chi_{2}}\right) \cong \begin{cases}V_{\epsilon^{m}}(-m) & \text { if } n=2 m \text { is even, } \\ V_{\chi_{2}} \otimes V_{\epsilon^{m}}(-m) & \text { if } n=2 m+1 \text { is odd. }\end{cases}
$$

We thus get the following corollary.

Corollary 2.4. Let $k$ be a perfect field of characteristic $p \neq 2$, let $f: \mathbb{A}_{k}^{n} \rightarrow \mathbb{A}_{k}^{1}$ be the $k$-morphism defined by

$$
f(z)=z_{1}^{2}+\cdots+z_{n}^{2}
$$

and let $R \Phi_{f}\left(\overline{\mathbb{Q}}_{\ell}\right)$ be the vanishing cycles complex of the morphism

$$
\mathbb{A}_{k}^{n} \times_{\mathbb{A}_{k}^{1}} \mathbb{A}_{(0)}^{1} \rightarrow \mathbb{A}_{(0)}^{1}
$$

induced from $f$ by base change. Then with the notation of 2.3, we have

$$
\left(R^{n-1} \Phi_{f}\left(\overline{\mathbb{Q}}_{\ell}\right)\right)_{0} \cong \begin{cases}V_{\epsilon^{m}}(-m) & \text { if } n=2 m \text { is even } \\ V_{\chi_{2}} \otimes V_{\epsilon^{m}}(-m) & \text { if } n=2 m+1 \text { is odd. }\end{cases}
$$

Note that the above formulas are compatible with those of $[5, \mathrm{XV} 2.2 .5 \mathrm{D}, \mathrm{E}]$ for algebraically closed field $k$. 


\section{Use of Laumon's local Fourier transformation}

The Artin-Schreier morphism

$$
\mathbb{A}_{k}^{1} \rightarrow \mathbb{A}_{k}^{1}, \quad t \mapsto t^{p}-t
$$

is a $\mathbb{Z} / p$-torsor. Fix a nontrivial additive character $\psi: \mathbb{Z} / p \rightarrow \overline{\mathbb{Q}}_{\ell}^{*}$. Pushing-forward the Artin-Schreier torsor using $\psi^{-1}$, we get a lisse sheaf $\mathscr{L}_{\psi}$ on $\mathbb{A}_{k}^{1}$. Denote the inverse image of $\mathscr{L}_{\psi}$ under the morphism

$$
\mathbb{A}_{k}^{1} \times_{k} \mathbb{A}_{k}^{1} \rightarrow \mathbb{A}_{k}^{1}, \quad\left(t, t^{\prime}\right) \rightarrow t t^{\prime}
$$

by $\mathscr{L}_{\psi}\left(t t^{\prime}\right)$. Let $\mathbb{A}_{k}^{1} \times_{k} \mathbb{A}_{k}^{1} \hookrightarrow \mathbb{A}_{k}^{1} \times_{k} \mathbb{P}_{k}^{1}$ be the open immersion defined by the canonical open immersion $\mathbb{A}_{k}^{1}=\mathbb{P}_{k}^{1}-\left\{\infty^{\prime}\right\} \hookrightarrow \mathbb{P}_{k}^{1}$. Denoted by $\overline{\mathscr{L}_{\psi}\left(t t^{\prime}\right)}$ the sheaf on $\mathbb{A}_{k}^{1} \times_{k} \mathbb{P}_{k}^{1}$ obtained from the sheaf $\mathscr{L}_{\psi}\left(t t^{\prime}\right)$ on $\mathbb{A}_{k}^{1} \times{ }_{k} \mathbb{A}_{k}^{1}$ by extension by zero. To distinguish the two factors in $\mathbb{A}_{k}^{1} \times_{k} \mathbb{A}_{k}^{1}$ and in $\mathbb{A}_{k}^{1} \times_{k} \mathbb{P}_{k}^{1}$, we denoted the objects related to the second factor by symbols with the superscript '. Denoted by $\mathbb{P}_{\left(\infty^{\prime}\right)}^{1}$ the Henselization of $\mathbb{P}_{k}^{1}$ at $\infty^{\prime}$, denote by $\eta_{\infty^{\prime}}$ its generic point, and denoted the restriction of $\overline{\mathscr{L}_{\psi}\left(t t^{\prime}\right)}$ to $\mathbb{A}_{(0)}^{1} \times_{k} \mathbb{P}_{\left(\infty^{\prime}\right)}^{1}$ also by $\overline{\mathscr{L}_{\psi}\left(t t^{\prime}\right)}$. Fixed a uniformizer $\pi$ of $S$. We have a $k$-morphism $S \rightarrow \mathbb{A}_{k}^{1}$ induced by the $k$-homomorphism

$$
k[t] \rightarrow \Gamma\left(S, \mathscr{O}_{S}\right), \quad t \mapsto \pi .
$$

It induces a $k$-morphism $S \rightarrow \mathbb{A}_{(0)}^{1}$, which we denote also by $\pi$. Denoted by $\overline{\mathscr{L}_{\psi}\left(\pi t^{\prime}\right)}$ the inverse image of $\overline{\mathscr{L}_{\psi}\left(t t^{\prime}\right)}$ under the morphism

$$
S \times{ }_{k} \mathbb{P}_{\left(\infty^{\prime}\right)}^{1} \stackrel{\pi \times \mathrm{id}_{\mathbb{P}_{(\infty)}^{1}}}{\rightarrow} \mathbb{A}_{(0)}^{1} \times_{k} \mathbb{P}_{\left(\infty^{\prime}\right)}^{1}
$$

Our proof of Theorem 2.2 relies on the following lemma.

Lemma 3.1. Let $g: Y \rightarrow S$ be a flat morphism of finite type, let $K \in \operatorname{ob} D_{c}^{b}\left(Y, \overline{\mathbb{Q}}_{\ell}\right)$, and let $y$ be a k-rational point in the special fiber $g^{-1}(s)$. Suppose that $Y$ is regular and pure of dimension $n,\left.g\right|_{Y-\{y\}}$ is smooth, and the sheaves $\left.\mathscr{H}^{q}(K)\right|_{Y-\{y\}}$ are lisse for all $q$.

(i) $R \Phi_{\eta_{\infty^{\prime}}}\left(p_{1}^{*} j_{!}\left(R \Phi_{g}(K)_{y}\right) \otimes^{L} \overline{\mathscr{L}_{\psi}\left(\pi t^{\prime}\right)}\right)$ is supported at $\left(s, \bar{\infty}^{\prime}\right)$, and $R \Phi_{\eta_{\infty^{\prime}}}$ $\left(\operatorname{pr}_{1}^{*} K \otimes^{L}\left(g \times \operatorname{id}_{\mathbb{P}_{\left(\infty^{\prime}\right)}^{1}}\right)^{*} \overline{\mathscr{L}_{\psi}\left(\pi t^{\prime}\right)}\right)$ is supported at $\left(y, \bar{\infty}^{\prime}\right)$, where $R \Phi_{g}(K)_{y}$ is a complex of $\operatorname{Gal}(\bar{\eta} / \eta)$-module and is regarded as an object in $D_{c}^{b}\left(\eta, \overline{\mathbb{Q}}_{\ell}\right)$, $j: \eta \hookrightarrow S$ is the canonical open immersion, $R \Phi_{\eta_{\infty^{\prime}}}$ denotes the vanishing cycles functors for the projections

$$
S \times{ }_{k} \mathbb{P}_{\left(\infty^{\prime}\right)}^{1} \rightarrow \mathbb{P}_{\left(\infty^{\prime}\right)}^{1}, \quad Y \times_{k} \mathbb{P}_{\left(\infty^{\prime}\right)}^{1} \rightarrow \mathbb{P}_{\left(\infty^{\prime}\right)}^{1},
$$

and $p_{1}, \mathrm{pr}_{1}$ are the projections

$$
p_{1}: S \times{ }_{k} \mathbb{P}_{\left(\infty^{\prime}\right)}^{1} \rightarrow S, \quad \operatorname{pr}_{1}: Y \times_{k} \mathbb{P}_{\left(\infty^{\prime}\right)}^{1} \rightarrow Y .
$$

(ii) We have a canonical isomorphism

$$
\begin{aligned}
& R \Phi_{\eta_{\infty^{\prime}}}\left(p_{1}^{*} j_{!}\left(R \Phi_{g}(K)_{y}\right) \otimes^{L} \overline{\mathscr{L}_{\psi}\left(\pi t^{\prime}\right)}\right)_{\left(s, \infty^{\prime}\right)} \\
& \quad \cong R \Phi_{\eta_{\infty^{\prime}}}\left(\operatorname{pr}_{1}^{*} K \otimes^{L}\left(g \times \operatorname{id}_{\mathbb{P}_{\left(\infty^{\prime}\right)}}\right)^{*} \overline{\mathscr{L}_{\psi}\left(\pi t^{\prime}\right)}\right)_{\left(y, \infty^{\prime}\right)} .
\end{aligned}
$$


(iii) Suppose $K$ is a lisse sheaf. Then $R^{i} \Phi_{\eta_{\infty^{\prime}}}\left(\operatorname{pr}_{1}^{*} K \otimes^{L}\left(g \times \operatorname{id}_{\mathbb{P}_{\left(\infty^{\prime}\right)}^{1}}\right)^{*} \overline{\mathscr{L}_{\psi}\left(\pi t^{\prime}\right)}\right)$ vanishes for $i \neq n$, and $R^{n} \Phi_{\eta_{\infty^{\prime}}}\left(\operatorname{pr}_{1}^{*} K \otimes^{L}\left(g \times \operatorname{id}_{\mathbb{P}_{\left(\infty^{\prime}\right)}^{1}}\right)^{*} \frac{\left(\infty^{\prime}\right)}{\mathscr{L}_{\psi}\left(\pi t^{\prime}\right)}\right)$ is a skyscraper sheaf on $Y \times_{k} \bar{\infty}^{\prime}$ supported at $\left(y, \bar{\infty}^{\prime}\right)$.

(iv) Under the condition of (iii), we have a canonical isomorphism of $\operatorname{Gal}\left(\bar{\eta}_{\infty^{\prime}} / \eta_{\infty^{\prime}}\right)$-modules

$$
\mathscr{F}^{\left(0, \infty^{\prime}\right)}\left(R^{n-1} \Phi_{g}(K)_{y}\right) \cong R^{n} \Phi_{\eta_{\infty^{\prime}}}\left(\operatorname{pr}_{1}^{*} K \otimes^{L}\left(g \times \operatorname{id}_{\mathbb{P}_{\left(\infty^{\prime}\right)}^{1}}\right)^{*} \overline{\mathscr{L}_{\psi}\left(\pi t^{\prime}\right)}\right)_{\left(y, \infty^{\prime}\right)}
$$

We will prove Lemma 3.1 in Section 4. Let us deduce Theorem 2.2 from Lemma 3.1 and the Künneth formula for the nearby cycles functor in $[9,4.7]$.

Proof of Theorem 2.2. Since $X_{i}(i=1,2)$ are regular and $k$ is perfect, $X_{i}$ are smooth over $k$, and hence $X=X_{1} \times_{k} X_{2}$ is smooth over $k$. It follows that $X$ is regular. Note that for any smooth $k$-scheme $Y$ and any $k$-morphism of finite type $f: Y \rightarrow \mathbb{A}_{k}^{1}, f$ is smooth at a $k$-point $y$ of $Y$, if and only if $d f(y) \in \Omega_{Y / k, y}^{1} \otimes_{\mathscr{O}_{Y}, y} k(y)$ is nonzero, where by abuse of notation, we denote also by $f$ the image of $t$ under the $k$-homomorphism

$$
k[t] \rightarrow \Gamma\left(Y, \mathscr{O}_{Y}\right)
$$

corresponding to the $k$-morphism $f$. This follows from $\left[8,17.11 .1(\mathrm{~b}) \Leftrightarrow\left(\mathrm{c}^{\prime}\right)\right]$.

Let us prove $\left.f\right|_{X-\{x\}}$ is smooth. By base change to an algebraic closure of $k$, we are reduced to the case where $k$ is algebraically closed. Suppose $x^{(0)}=\left(x_{1}^{(0)}, x_{2}^{(0)}\right)$ is a $k$-rational point in $X=X_{1} \times_{k} X_{2}$, where $f$ is not smooth. By our assumption, $f$ is smooth on $\left(X_{1}-\left\{x_{1}\right\}\right) \times\left(X_{2}-\left\{x_{2}\right\}\right)$. It follows that either $x_{1}^{(0)}=x_{1}$ or $x_{2}^{(0)}=$ $x_{2}$. Without the loss of generality, assume $x_{1}^{(0)}=x_{1}$. If $x_{2}^{(0)} \neq x_{2}$, then by our assumption, $f_{2}$ is smooth at $x_{2}^{(0)}$. By the discussion above, $d f_{2}$ defines a nonzero element in $\Omega_{X_{2} / k, x_{2}^{(0)}}^{1} \otimes_{\mathscr{O}_{X_{2}, x_{2}^{(0)}}} k\left(x_{2}^{(0)}\right)$. Under the identification

$$
\Omega_{X / k}^{1} \cong p_{1}^{*} \Omega_{X_{1} / k}^{1} \bigoplus p_{2}^{*} \Omega_{X_{2} / k}^{1},
$$

where $p_{i}: X_{1} \times_{k} X_{2} \rightarrow X_{i}(i=1,2)$ are the projections, $d f$ is identified with $\left(d f_{1}, d f_{2}\right)$. It follows that $d f$ defines a nonzero element in $\Omega_{X / k, x^{(0)}}^{1} \otimes_{\mathscr{O}_{X, x}(0)} k\left(x^{(0)}\right)$. So $f$ is smooth at $x^{(0)}$. Contradiction. So we must have $x_{2}^{(0)}=x_{2}$ and hence, $x^{(0)}=x$. This proves (i).

By [3, Sommes trig. 1.3.1], we have a canonical isomorphism

$$
\left(f_{1} \times \operatorname{id}_{\mathbb{P}_{k}^{1}}\right)^{*} \overline{\mathscr{L}_{\psi}\left(t t^{\prime}\right)} \otimes^{L}\left(f_{2} \times \operatorname{id}_{\mathbb{P}_{k}^{1}}\right)^{*} \overline{\mathscr{L}_{\psi}\left(t t^{\prime}\right)} \cong\left(f \times \operatorname{id}_{\mathbb{P}_{k}^{1}}\right)^{*} \overline{\mathscr{L}_{\psi}\left(t t^{\prime}\right)},
$$

where the external tensor product is taken with respect to the Cartesian diagram

$$
\begin{array}{ccc}
X_{1} \times_{k} X_{2} \times_{k} \mathbb{P}_{k}^{1} & \rightarrow & X_{2} \times_{k} \mathbb{P}_{k}^{1}, \\
\downarrow & & \downarrow \\
X_{1} \times_{k} \mathbb{P}_{k}^{1} & \rightarrow & \mathbb{P}_{k}^{1} .
\end{array}
$$

So we have

$$
\begin{aligned}
\left(\operatorname{pr}_{X_{1}}^{*} K_{1} \otimes^{L}\left(f_{1} \times \operatorname{id}_{\mathbb{P}_{k}^{1}}\right)^{*} \overline{\mathscr{L}_{\psi}\left(t t^{\prime}\right)}\right) & \otimes^{L}\left(\operatorname{pr}_{X_{2}}^{*} K_{2} \otimes^{L}\left(f_{2} \times \operatorname{id}_{\mathbb{P}_{k}^{1}}\right)^{*} \overline{\mathscr{L}_{\psi}\left(t t^{\prime}\right)}\right) \\
& \cong \operatorname{pr}_{X}^{*} K \otimes^{L}\left(f \times \operatorname{id}_{\mathbb{P}_{k}^{1}}\right)^{*} \overline{\mathscr{L}_{\psi}\left(t t^{\prime}\right)}
\end{aligned}
$$


where

$\operatorname{pr}_{X}: X_{1} \times_{k} X_{2} \times_{k} \mathbb{P}_{k}^{1} \rightarrow X_{1} \times_{k} X_{2}, \quad \operatorname{pr}_{X_{1}}: X_{1} \times_{k} \mathbb{P}_{k}^{1} \rightarrow X_{1}, \quad \operatorname{pr}_{X_{2}}: X_{2} \times_{k} \mathbb{P}_{k}^{1} \rightarrow X_{2}$ are the projections. Therefore, we deduce from [9, 4.7] a canonical isomorphism

$$
\begin{aligned}
& R \Psi_{\eta_{\infty^{\prime}}}\left(\operatorname{pr}_{X_{1}}^{*} K_{1} \otimes^{L}\left(f_{1} \times \operatorname{id}_{\mathbb{P}_{k}^{1}}\right)^{*} \overline{\mathscr{L}_{\psi}\left(t t^{\prime}\right)}\right) \otimes^{L} R \Psi_{\eta_{\infty^{\prime}}}\left(\operatorname{pr}_{X_{2}}^{*} K_{2} \otimes^{L}\left(f_{2} \times \operatorname{id}_{\mathbb{P}_{k}^{1}}\right)^{*} \overline{\mathscr{L}_{\psi}\left(t t^{\prime}\right)}\right) \\
& \cong R \Psi_{\eta_{\infty^{\prime}}}\left(\operatorname{pr}_{X}^{*} K \otimes^{L}\left(f \times \operatorname{id}_{\mathbb{P}_{k}^{1}}\right)^{*} \overline{\mathscr{L}_{\psi}\left(t t^{\prime}\right)}\right) .
\end{aligned}
$$

Since $\operatorname{pr}_{X}^{*} K \otimes{ }^{L}\left(f \times \mathrm{id}_{\mathbb{P}_{k}^{1}}\right)^{*} \overline{\mathscr{L}_{\psi}\left(t t^{\prime}\right)}$ vanishes on $X \times_{k} \bar{\infty}^{\prime}$, we have

$$
R \Phi_{\eta_{\infty^{\prime}}}\left(\operatorname{pr}_{X}^{*} K \otimes^{L}\left(f \times \operatorname{id}_{\mathbb{P}_{k}^{1}}\right)^{*} \overline{\mathscr{L}_{\psi}\left(t t^{\prime}\right)}\right) \cong R \Psi_{\eta_{\infty^{\prime}}}\left(\operatorname{pr}_{X}^{*} K \otimes^{L}\left(f \times \operatorname{id}_{\mathbb{P}_{k}^{1}}\right)^{*} \overline{\mathscr{L}_{\psi}\left(t t^{\prime}\right)}\right),
$$

and we have similar isomorphisms if we replace $f$ by $f_{i}$ and $\operatorname{pr}_{X}^{*} K$ by $\operatorname{pr}_{X_{i}}^{*} K_{i}$. So we have a canonical isomorphism

$$
\begin{aligned}
& R \Phi_{\eta_{\infty^{\prime}}}\left(\operatorname{pr}_{X_{1}}^{*} K_{1} \otimes^{L}\left(f_{1} \times \operatorname{id}_{\mathbb{P}_{k}^{1}}\right)^{*} \overline{\mathscr{L}_{\psi}\left(t t^{\prime}\right)}\right) \otimes^{L} \\
& R \Phi_{\eta_{\infty^{\prime}}}\left(\operatorname{pr}_{X_{2}}^{*} K_{2} \otimes^{L}\left(f_{2} \times \operatorname{id}_{\mathbb{P}_{k}^{1}}\right)^{*} \overline{\mathscr{L}_{\psi}\left(t t^{\prime}\right)}\right) \\
& \cong R \Phi_{\eta_{\infty^{\prime}}}\left(\operatorname{pr}_{X}^{*} K \otimes^{L}\left(f \times \mathrm{id}_{\mathbb{P}_{k}^{1}}\right)^{*} \overline{\mathscr{L}_{\psi}\left(t t^{\prime}\right)}\right) .
\end{aligned}
$$

Combined with Lemma 3.1(i), we get

$$
\begin{gathered}
R \Phi_{\eta_{\infty^{\prime}}}\left(\operatorname{pr}_{X_{1}}^{*} K_{1} \otimes^{L}\left(f_{1} \times \mathrm{id}_{\mathbb{P}_{k}^{1}}\right)^{*} \overline{\mathscr{L}_{\psi}\left(t t^{\prime}\right)}\right)_{\left(x_{1}, \infty^{\prime}\right)} \otimes^{L} \\
R \Phi_{\eta_{\infty^{\prime}}}\left(\operatorname{pr}_{X_{2}}^{*} K_{2} \otimes^{L}\left(f_{2} \times \mathrm{id}_{\mathbb{P}_{k}^{1}}\right)^{*} \overline{\mathscr{L}_{\psi}\left(t t^{\prime}\right)}\right)_{\left(x_{2}, \infty^{\prime}\right)} \\
\cong R \Phi_{\eta_{\infty^{\prime}}}\left(\operatorname{pr}_{X}^{*} K \otimes^{L}\left(f \times \mathrm{id}_{\mathbb{P}_{k}^{1}}\right)^{*} \overline{\mathscr{L}_{\psi}\left(t t^{\prime}\right)}\right)_{\left(x, \infty^{\prime}\right)} .
\end{gathered}
$$

By Lemma 3.1(ii), this induces a canonical isomorphism

$$
\begin{aligned}
& R \Phi_{\eta_{\infty^{\prime}}}\left(p_{1}^{*} j_{!}\left(R \Phi_{f_{1}}\left(K_{1}\right)_{x_{1}}\right) \otimes^{L} \overline{\mathscr{L}_{\psi}\left(t t^{\prime}\right)}\right)_{\left(0, \infty^{\prime}\right)} \otimes^{L} \\
& R \Phi_{\eta_{\infty^{\prime}}}\left(p_{1}^{*} j_{!}\left(R \Phi_{f_{2}}\left(K_{2}\right)_{x}\right) \otimes^{L} \overline{\mathscr{L}_{\psi}\left(t t^{\prime}\right)}\right)_{\left(0, \infty^{\prime}\right)} \\
& \cong R \Phi_{\eta_{\infty^{\prime}}}\left(p_{1}^{*} j_{!}\left(R \Phi_{f}(K)_{x}\right) \otimes^{L} \overline{\mathscr{L}_{\psi}\left(t t^{\prime}\right)}\right)_{\left(0, \infty^{\prime}\right)},
\end{aligned}
$$

and under the assumption of Theorem 2.2(iii), we get a canonical isomorphism

$$
\begin{aligned}
\mathscr{F}^{\left(0, \infty^{\prime}\right)}\left(R^{n_{1}-1} \Phi_{f_{1}}\left(K_{1}\right)_{x_{1}}\right) & \otimes \mathscr{F}^{\left(0, \infty^{\prime}\right)}\left(R^{n_{2}-1} \Phi_{f_{2}}\left(K_{2}\right)_{x_{2}}\right) \\
& \cong \mathscr{F}^{\left(0, \infty^{\prime}\right)}\left(R^{n-1} \Phi_{f}(K)_{x}\right) .
\end{aligned}
$$

By $[12,2.7 .2 .2(\mathrm{i})]$ and the inversion formula for local Fourier transformation $[12,2.4 .3$ (i)c)], under the assumption of Theorem 2.2(iii), we have a canonical isomorphism

$$
R^{n_{1}-1} \Phi_{f_{1}}\left(K_{1}\right)_{x_{1}} * R^{n_{2}-1} \Phi_{f_{2}}\left(K_{2}\right)_{x_{2}} \cong R^{n-1} \Phi_{f}(K)_{x}
$$

The argument in the proof of various results in [12] also shows that in general we have a canonical isomorphism

$$
R \Phi_{f_{1}}\left(K_{1}\right)_{x_{1}} * R \Phi_{f_{2}}\left(K_{2}\right)_{x_{2}} \cong R \Phi_{f}(K)_{x}
$$


We give a detailed argument for completeness. Choose $L, L_{1}, L_{2} \in$ ob $D_{c}^{b}\left(\mathbb{A}_{k}^{1}-\{0\}, \overline{\mathbb{Q}}_{\ell}\right)$ such that

$$
\left.L\right|_{\eta_{0}} \cong R \Phi_{f}(K)_{x},\left.\quad L_{1}\right|_{\eta_{0}} \cong R \Phi_{f_{1}}\left(K_{1}\right)_{x_{1}},\left.\quad L_{2}\right|_{\eta_{0}} \cong R \Phi_{f_{2}}\left(K_{2}\right)_{x_{2}},
$$

and such that $\mathscr{H}^{q}(L), \mathscr{H}^{q}\left(L_{1}\right), \mathscr{H}^{q}\left(L_{2}\right)$ are lisse on $\mathbb{A}_{k}^{1}-\{0\}$ and tamely ramified at $\infty$ for all $q$. The existence of $L, L_{1}$, and $L_{2}$ follows from Lemma 3.2 below. Let

$$
\mathscr{F}: D_{c}^{b}\left(\mathbb{A}_{k}^{1}, \overline{\mathbb{Q}}_{\ell}\right) \rightarrow D_{c}^{b}\left(\mathbb{A}_{k}^{1}, \overline{\mathbb{Q}}_{\ell}\right)
$$

be the Deligne-Fourier transformation $([12,1.2 .1 .1])$, and let $\iota: \mathbb{A}_{k}^{1}-\{0\} \hookrightarrow \mathbb{A}_{k}^{1}$ be the canonical open immersion. By the same argument as the proof of $[12,2.3 .3 .1]$ (which relies on $[12,1.3 .1 .2])$, by the fact that $\mathscr{H}^{q}(L)$ and $\mathscr{H}^{q}\left(L_{i}\right)$ are lisse on $\mathbb{A}_{k}^{1}-\{0\}$ and tamely ramified at $\infty$, and by $[12,2.4 .3$ (iii)b)], we have

$$
\begin{aligned}
& \left.\mathscr{F}(\iota ! L)[-1]\right|_{\eta_{\infty^{\prime}}} \cong R \Phi_{\eta_{\infty^{\prime}}}\left(p_{1}^{*} j_{!}\left(R \Phi_{f}(K)_{x}\right) \otimes^{L} \overline{\mathscr{L}_{\psi}\left(t t^{\prime}\right)}\right)_{\left(0, \infty^{\prime}\right)}, \\
& \left.\mathscr{F}\left(\iota ! L_{1}\right)[-1]\right|_{\eta_{\infty^{\prime}}} \cong R \Phi_{\eta_{\infty^{\prime}}}\left(p_{1}^{*} j_{!}\left(R \Phi_{f_{1}}\left(K_{1}\right)_{x_{1}}\right) \otimes^{L} \overline{\mathscr{L}_{\psi}\left(t t^{\prime}\right)}\right)_{\left(0, \infty^{\prime}\right)}, \\
& \left.\mathscr{F}\left(\iota_{!} L_{2}\right)[-1]\right|_{\eta_{\infty^{\prime}}} \cong R \Phi_{\eta_{\infty^{\prime}}}\left(p_{1}^{*} j_{!}\left(R \Phi_{f_{2}}\left(K_{2}\right)_{x_{2}}\right) \otimes^{L} \overline{\mathscr{L}_{\psi}\left(t t^{\prime}\right)}\right)_{\left(0, \infty^{\prime}\right)} .
\end{aligned}
$$

So we can write the isomorphism (3.2) as

$$
\left.\left.\left(\mathscr{F}\left(\iota ! L_{1}\right) \otimes^{L} \mathscr{F}\left(\iota ! L_{2}\right)\right)[-2]\right|_{\eta_{\infty^{\prime}}} \cong \mathscr{F}(\iota ! L)[-1]\right|_{\eta_{\infty^{\prime}}} .
$$

By $[12,1.2 .2 .7]$, we get the isomorphism

$$
\left.\left.\mathscr{F}\left(\iota ! L_{1} * \iota ! L_{2}\right)\right|_{\eta_{\infty^{\prime}}} \cong \mathscr{F}(\iota ! L)\right|_{\eta_{\infty^{\prime}}},
$$

where $\iota_{!} L_{1} * \iota ! L_{2}$ is the (global) convolution product of $\iota ! L_{1}$ and $\iota_{!} L_{2}$ ([12, 1.2.2.6]). By the above isomorphism and Lemma 3.3 below, we have

$$
R \Phi_{\eta_{0}}\left(\iota_{!} L_{1} * \iota_{!} L_{2}\right) \cong R \Phi_{\eta_{0}}(\iota ! L)
$$

where $R \Phi_{\eta_{0}}$ denotes the vanishing cycles functor relative to the identity morphism on $\mathbb{A}_{(0)}^{1}$. By $[12,2.7 .1 .1$ (iii)], this last isomorphism is exactly

$$
R \Phi_{f_{1}}\left(K_{1}\right)_{x_{1}} * R \Phi_{f_{2}}\left(K_{2}\right)_{x_{2}} \cong R \Phi_{f}(K)_{x} .
$$

Lemma 3.2. For any object $K$ in $D_{c}^{b}\left(\eta_{0}, \overline{\mathbb{Q}}_{\ell}\right)$, there exists an object $\bar{K}$ in $D_{c}^{b}\left(\mathbb{A}_{k}^{1}-\right.$ $\left.\{0\}, \overline{\mathbb{Q}}_{\ell}\right)$ such that $\left.\bar{K}\right|_{\eta_{0}} \cong K$ and such that $\mathscr{H}^{q}(\bar{K})$ are lisse on $\mathbb{A}_{k}^{1}-\{0\}$ and tamely ramified at $\infty$ for all $q$.

Proof. By the description of the derived category of $\ell$-adic sheaves in $[4,1.1]$, there exists a finite extension $E$ of $\mathbb{Q}_{\ell}$ such that $K$ is given by an object in $D_{c}^{b}\left(\eta_{0}, R\right)$, where $R$ is the integer ring of $E$. Let $\lambda$ be a uniformizer of $R$. The object $K$ corresponds to a projective system defined by complexes $K_{n} \in \mathrm{ob} D_{c t f}\left(\eta_{0}, R /\left(\lambda^{n}\right)\right)$ and isomorphisms

$$
K_{n+1} \otimes_{R /\left(\lambda^{n+1}\right)}^{L} R /\left(\lambda^{n}\right) \cong K_{n}
$$

in $D_{c t f}\left(\eta_{0}, R /\left(\lambda^{n}\right)\right)$. By [3, Rapport 4.6], we can represent each $K_{n}$ by a bounded complex, so that all components $K_{n}^{i}(i \in \mathbb{Z})$ are constructible flat sheaves of $R /\left(\lambda^{n}\right)$ modules on $\eta_{0}$. As $\eta_{0}$ is the spectrum of a field, a flat constructible sheaf of $R /\left(\lambda^{n}\right)$-modules is just a free $R /\left(\lambda^{n}\right)$-module of finite rank with a continuous $\operatorname{Gal}\left(\bar{\eta}_{0} / \eta_{0}\right)$-action. Denote $\mathbb{A}_{k}^{1}-\{0\}$ by $\mathbb{G}_{m, k}$, and let $\pi_{1}\left(\mathbb{G}_{m, k}, \bar{\eta}_{0}\right)^{\text {tame, } \infty}$ be the 
quotient of $\pi_{1}\left(\mathbb{G}_{m, k}, \bar{\eta}_{0}\right)$ classifying finite etale coverings of $\mathbb{G}_{m, k}$ tamely ramified at $\infty$. By $[12,2.2 .2 .2]$, the composite of the canonical homomorphisms

$$
\operatorname{Gal}\left(\bar{\eta}_{0} / \eta_{0}\right)=\pi_{1}\left(\eta_{0}, \bar{\eta}_{0}\right) \rightarrow \pi_{1}\left(\mathbb{G}_{m, k}, \bar{\eta}_{0}\right) \rightarrow \pi_{1}\left(\mathbb{G}_{m, k}, \bar{\eta}_{0}\right)^{\text {tame }, \infty}
$$

has a retraction

$$
r: \pi_{1}\left(\mathbb{G}_{m, k}, \bar{\eta}_{0}\right)^{\operatorname{tame}, \infty} \rightarrow \operatorname{Gal}\left(\bar{\eta}_{0} / \eta_{0}\right) .
$$

Through the composite

$$
\pi_{1}\left(\mathbb{G}_{m, k}, \bar{\eta}_{0}\right) \rightarrow \pi_{1}\left(\mathbb{G}_{m, k}, \bar{\eta}_{0}\right)^{\text {tame }, \infty} \stackrel{r}{\rightarrow} \operatorname{Gal}\left(\bar{\eta}_{0} / \eta_{0}\right),
$$

each free $R /\left(\lambda^{n}\right)$-module of finite rank with a continuous $\operatorname{Gal}\left(\bar{\eta}_{0} / \eta_{0}\right)$-action can be endowed with a continuous $\pi_{1}\left(\mathbb{G}_{m, k}, \bar{\eta}_{0}\right)$-action extending the given action of $\operatorname{Gal}\left(\bar{\eta}_{0} / \eta_{0}\right)$ at 0 and tamely ramified at $\infty$. In particular, each component $K_{n}^{i}$ of the complex $K_{n}$ now becomes a free $R /\left(\lambda^{n}\right)$-module of finite rank with continuous $\pi_{1}\left(\mathbb{G}_{m, k}, \bar{\eta}_{0}\right)$-action extending the given action of $\operatorname{Gal}\left(\bar{\eta}_{0} / \eta_{0}\right)$ at 0 and tamely ramified at $\infty$. We thus get a lisse constructible flat sheaf $\bar{K}_{n}^{i}$ of $R /\left(\lambda^{n}\right)$-modules on $\mathbb{G}_{m, k}$ tamely ramified at $\infty$ with the property $\left.\bar{K}_{n}^{i}\right|_{\eta_{0}}=K_{n}^{i}$. The differential morphisms $d_{K_{n}}^{i}: K_{n}^{i} \rightarrow K_{n}^{i+1}$ for the complex $K_{n}$ are linear maps compatible with the $\operatorname{Gal}\left(\bar{\eta}_{0} / \eta_{0}\right)$-action, and induce linear maps compatible with the $\pi_{1}\left(\mathbb{G}_{m, k}, \bar{\eta}_{0}\right)$-action. So they define morphisms of sheaves $d_{\bar{K}_{n}}^{i}: \bar{K}_{n}^{i} \rightarrow \bar{K}_{n}^{i+1}$ with the property $d \circ d=0$. We thus get a complex $\bar{K}_{n} \in \operatorname{ob} D_{c t f}\left(\mathbb{G}_{m, k}, R /\left(\lambda^{n}\right)\right)$ with the property $\left.\bar{K}_{n}\right|_{\eta_{0}} \cong K_{n}$. Finally, let us prove the isomorphisms

$$
K_{n+1} \otimes_{R /\left(\lambda^{n+1}\right)}^{L} R /\left(\lambda^{n}\right) \cong K_{n}
$$

in $D_{c t f}\left(\eta_{0}, R /\left(\lambda^{n}\right)\right)$ can be extended to isomorphisms

$$
\bar{K}_{n+1} \otimes_{R /\left(\lambda^{n+1}\right)}^{L} R /\left(\lambda^{n}\right) \cong \bar{K}_{n}
$$

in $D_{c t f}\left(\mathbb{G}_{m, k}, R /\left(\lambda^{n}\right)\right)$. The projective system $\left(\bar{K}_{n}\right) \in$ ob $D_{c}^{b}\left(\mathbb{G}_{m, k}, R\right)$ then defines an object $\bar{K}$ in ob $D_{c}^{b}\left(\mathbb{G}_{m, k}, \overline{\mathbb{Q}}_{\ell}\right)$ with the required property. Since components of $K_{n+1}$ are free $R /\left(\lambda^{n+1}\right)$-modules of finite rank, we have

$$
K_{n+1} \otimes_{R /\left(\lambda^{n+1}\right)}^{L} R /\left(\lambda^{n}\right) \cong K_{n+1} \otimes_{R /\left(\lambda^{n+1}\right)} R /\left(\lambda^{n}\right) .
$$

The isomorphism $K_{n+1} \otimes_{R /\left(\lambda^{n+1}\right)}^{L} R /\left(\lambda^{n}\right) \cong K_{n}$ in $D_{c t f}\left(\eta_{0}, R /\left(\lambda^{n}\right)\right)$ can be represented by a diagram

$$
\begin{aligned}
L & \\
K_{n+1} \otimes_{R /\left(\lambda^{n+1}\right)} R /\left(\lambda^{n}\right) & \searrow K_{n},
\end{aligned}
$$

where the two arrows are quasi-isomorphisms. By [3, Rapport 4.7], we may assume that $L$ is a bounded above complex of free $R /\left(\lambda^{n}\right)$-modules of finite rank with continuous $\operatorname{Gal}\left(\bar{\eta}_{0} / \eta_{0}\right)$-action. Again through the composite

$$
\pi_{1}\left(\mathbb{G}_{m, k}, \bar{\eta}_{0}\right) \rightarrow \pi_{1}\left(\mathbb{G}_{m, k}, \bar{\eta}_{0}\right)^{\text {tame }, \infty} \stackrel{r}{\rightarrow} \operatorname{Gal}\left(\bar{\eta}_{0} / \eta_{0}\right),
$$

we can extend $L$ to a complex $\bar{L}$ of lisse constructible flat sheaves of $R /\left(\lambda^{n}\right)$-modules on $\mathbb{G}_{m, k}$ and extend the above diagram to a diagram

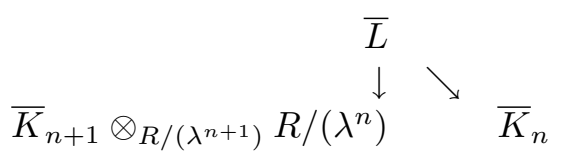


of complexes of lisse sheaves of $\mathbb{G}_{m, k}$ so that the two arrows are quasi-isomorphisms. This defines the isomorphism

$$
\bar{K}_{n+1} \otimes_{R /\left(\lambda^{n+1}\right)} R /\left(\lambda^{n}\right) \cong \bar{K}_{n}
$$

in $D_{c t f}\left(\mathbb{G}_{m, k}, R /\left(\lambda^{n}\right)\right)$ that we are seeking.

Lemma 3.3. Let $L_{1}, L_{2} \in$ ob $D_{c}^{b}\left(\mathbb{A}_{k}^{1}, \overline{\mathbb{Q}}_{\ell}\right)$. If $\left.\left.\mathscr{F}\left(L_{1}\right)\right|_{\eta_{\infty^{\prime}}} \cong \mathscr{F}\left(L_{2}\right)\right|_{\eta_{\infty^{\prime}}}$, then $R \Phi_{\eta_{0}}\left(L_{1}\right) \cong R \Phi_{\eta_{0}}\left(L_{2}\right)$.

Proof. Let $L \in$ ob $D_{c}^{b}\left(\mathbb{A}_{k}^{1}, \overline{\mathbb{Q}}_{\ell}\right)$ and let $L^{\prime}=\mathscr{F}(L)$. By the inversion formula for the Deligne-Fourier transformation $[12,1.2 .2 .1]$, we have

$$
\mathscr{F}^{\prime}\left(L^{\prime}\right)=b_{*} L(-1),
$$

where $b: \mathbb{A}_{k}^{1} \rightarrow \mathbb{A}_{k}^{1}$ is the morphism defined by $t \mapsto-t$, and $\mathscr{F}^{\prime}$ denote the DeligneFourier transformation for the dual of $\mathbb{A}_{k}^{1}$ (which is the second factor of $\mathbb{A}_{k}^{1} \times{ }_{k} \mathbb{A}_{k}^{1}$ ). So we have

$$
R \Phi_{\eta_{0}}(L)=b^{*} R \Phi_{\eta_{0}}\left(\mathscr{F}^{\prime}\left(L^{\prime}\right)\right)(1) .
$$

By $[12,2.3 .2 .1(\mathrm{i})]$ (which relies on $\left[3\right.$, Th. finitude 2.16]), $R \Phi_{\eta_{0}}\left(\mathscr{F}^{\prime}\left(L^{\prime}\right)\right)$ depends only on $\left.L^{\prime}\right|_{\eta_{\infty^{\prime}}}=\left.\mathscr{F}(L)\right|_{\eta_{\infty^{\prime}}}$. Our assertion follows.

Remark 3.4. We expect that Theorem 2.2 still holds, if we just assume that $\left.\mathscr{H}^{q}\left(K_{i}\right)\right|_{X_{i}-\left\{x_{i}\right\}}$ are lisse for all $q$. The difficulty is that we do not know whether this weaker condition implies that $R \Phi_{f}(K)$ is supported at $x$. Using Lemma 2.1 (i) and the canonical isomorphism $(3.1)$, one can show that $R \Phi_{\eta_{\infty^{\prime}}}\left(\operatorname{pr}_{X}^{*} K \otimes^{L}\left(f \times \mathrm{id}_{\mathbb{P}_{k}^{1}}\right)^{*} \overline{\mathscr{L}_{\psi}\left(t t^{\prime}\right)}\right)$ is supported at $\left(x, \infty^{\prime}\right)$ under this condition.

\section{Proof of Lemma 3.1}

We first prove Lemma 3.1(i) and (iii).

Proof of Lemma 3.1(i) and (iii). That $R \Phi_{\eta_{\infty^{\prime}}}\left(p_{1}^{*} j_{!}\left(R \Phi_{g}(K)_{y}\right) \otimes^{L} \overline{\mathscr{L}_{\psi}\left(\pi t^{\prime}\right)}\right)$ is supported at $\left(s, \bar{\infty}^{\prime}\right)$ follows from $[12,2.4 .2 .2]$ and the fact that the functor $R \Phi_{\eta_{\infty^{\prime}}}\left(p_{1}^{*} j_{!}(-) \otimes^{L} \overline{\mathscr{L}_{\psi}\left(\pi t^{\prime}\right)}\right)$ is exact. Here $1 / t^{\prime}$ is a uniformizing parameter $\pi^{\prime}$ on $\mathbb{P}_{\left(\infty^{\prime}\right)}^{1}$, and in Laumon's notation, $\overline{\mathscr{L}_{\psi}\left(\pi t^{\prime}\right)}$ is $\overline{\mathscr{L}}_{\psi}\left(\pi / \pi^{\prime}\right)$. By $[12,1.3 .1 .2], R \Phi_{\eta_{\infty^{\prime}}}\left(\overline{\mathscr{L}_{\psi}\left(\pi t^{\prime}\right)}\right)$ vanishes on $S \times_{k} \bar{\infty}^{\prime}$. On the other hand, $g \times \operatorname{id}_{\mathbb{P}_{\left(\infty^{\prime}\right)}^{1}}$ is smooth on $(Y-\{y\}) \times_{k} \mathbb{P}_{\left(\infty^{\prime}\right)}^{1}$, and $\left.\mathscr{H}^{q}(K)\right|_{Y-\{y\}}$ are lisse for all $q$. By the smooth base change theorem and the projection formula, we have

$$
\begin{aligned}
& \left.R \Phi_{\eta_{\infty^{\prime}}}\left(\operatorname{pr}_{1}^{*} K \otimes^{L}\left(g \times \operatorname{id}_{\mathbb{P}_{\left(\infty^{\prime}\right)}}\right)^{*} \overline{\mathscr{L}_{\psi}\left(\pi t^{\prime}\right)}\right)\right|_{(Y-\{y\}) \times_{k} \bar{\infty}^{\prime}} \\
& \left.\left.\quad \cong\left(\operatorname{pr}_{1}^{*} K\right)\right|_{(Y-\{y\}) \times{ }_{k} \bar{\infty}^{\prime}} \otimes^{L}\left(\left(g \times \mathrm{id}_{\bar{\infty}^{\prime}}\right)^{*} R \Phi_{\eta_{\infty^{\prime}}}\left(\overline{\mathscr{L}_{\psi}\left(\pi t^{\prime}\right)}\right)\right)\right|_{(Y-\{y\}) \times_{k} \bar{\infty}^{\prime}} \\
& \quad=0 .
\end{aligned}
$$

It follows that $R \Phi_{\eta_{\infty^{\prime}}}\left(\operatorname{pr}_{1}^{*} K \otimes^{L}\left(g \times \operatorname{id}_{\mathbb{P}_{\left(\infty^{\prime}\right)}^{1}}\right)^{*} \overline{\mathscr{L}_{\psi}\left(\pi t^{\prime}\right)}\right)$ is supported at $\left(y, \bar{\infty}^{\prime}\right)$. This proves Lemma 3.1(i).

Note that the restriction of $\overline{\mathscr{L}_{\psi}\left(\pi t^{\prime}\right)}$ to $S \times_{k} \eta_{\infty^{\prime}}$ is a lisse sheaf. So under the assumption of (iii), the restriction of $\operatorname{pr}_{1}^{*} K \otimes^{L}\left(g \times \operatorname{id}_{\mathbb{P}_{\left(\infty^{\prime}\right)}^{1}}\right)^{*} \overline{\mathscr{L}_{\psi}\left(\pi t^{\prime}\right)}[n]$ to $Y \times_{k} \eta_{\infty^{\prime}}$ 
is perverse. By $[9,4.5], R \Psi_{\eta_{\infty^{\prime}}}\left(\operatorname{pr}_{1}^{*} K \otimes^{L}\left(g \times \operatorname{id}_{\mathbb{P}_{\left(\infty^{\prime}\right)}^{1}}\right)^{*} \overline{\mathscr{L}_{\psi}\left(\pi t^{\prime}\right)}\right)[n]$ is perverse on

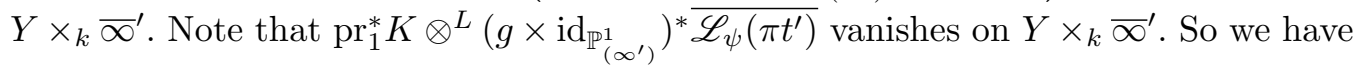
$R \Phi_{\eta_{\infty^{\prime}}}\left(\operatorname{pr}_{1}^{*} K \otimes^{L}\left(g \times \operatorname{id}_{\mathbb{P}_{\left(\infty^{\prime}\right)}^{1}}\right)^{*} \overline{\mathscr{L}_{\psi}\left(\pi t^{\prime}\right)}\right) \cong R \Psi_{\eta_{\infty^{\prime}}}\left(\operatorname{pr}_{1}^{*} K \otimes^{L}\left(g \times \operatorname{id}_{\mathbb{P}_{\left(\infty^{\prime}\right)}^{1}}\right)^{*} \overline{\mathscr{L}_{\psi}\left(\pi t^{\prime}\right)}\right)$. Hence $R \Phi_{\eta_{\infty^{\prime}}}\left(\operatorname{pr}_{1}^{*} K \otimes^{L}\left(g \times \operatorname{id}_{\mathbb{P}_{\left(\infty^{\prime}\right)}^{1}}\right)^{*} \overline{\mathscr{L}_{\psi}\left(\pi t^{\prime}\right)}\right)[n]$ is a perverse sheaf on $Y \times_{k} \bar{\infty}^{\prime}$ supported at $\left(y, \bar{\infty}^{\prime}\right)$. Lemma 3.1(iii) then follows.

Lemma 4.1. Let $K$ be an object in $D_{c}^{b}\left(S, \overline{\mathbb{Q}}_{\ell}\right)$, let

$$
p_{1}: S \times_{k} \mathbb{P}_{\left(\infty^{\prime}\right)}^{1} \rightarrow S, \quad p_{2}: S \times_{k} \mathbb{P}_{\left(\infty^{\prime}\right)}^{1} \rightarrow \mathbb{P}_{\left(\infty^{\prime}\right)}^{1}
$$

be the projections, and let $j: \eta \hookrightarrow S$ be the canonical open immersion. We have a canonical isomorphism

$$
R \Phi_{\eta_{\infty^{\prime}}}\left(p_{1}^{*} K \otimes^{L} \overline{\mathscr{L}_{\psi}\left(\pi t^{\prime}\right)}\right) \cong R \Phi_{\eta_{\infty^{\prime}}}\left(p_{1}^{*}\left(j ! R \Phi_{\eta}(K)\right) \otimes^{L} \overline{\mathscr{L}_{\psi}\left(\pi t^{\prime}\right)}\right)
$$

where $R \Phi_{\eta_{\infty}}$ denotes the vanishing cycles functor with respect to the projection $p_{2}$, $R \Phi_{\eta}(K)$ denotes the vanishing cycles complex of $K$ relative to $\mathrm{id}_{S}$, which is a complex of $\operatorname{Gal}(\bar{\eta} / \eta)$-modules and is regarded as an object in $D_{c}^{b}\left(\eta, \overline{\mathbb{Q}}_{\ell}\right)$, and can be identified with the cone of the specialization morphism $K_{\bar{s}} \rightarrow K_{\bar{\eta}}$.

Proof. Let $E$ be a finite extension of $\mathbb{Q}_{\ell}$ in $\overline{\mathbb{Q}}_{\ell}$ containing $\operatorname{im}(\psi)$, let $R$ be the integral closure of $\mathbb{Z}_{\ell}$ in $E$, and let $\lambda$ be a uniformizer of $R$. It suffices to prove the same assertion for any complex $K$ of sheaves of $R /\left(\lambda^{m}\right)$-modules for any positive integer $m$. For convenience, write $\Lambda$ for $R /\left(\lambda^{m}\right)$. For any scheme $X$, denote by $C(X, \Lambda)$ the triangulated category of complexes of etale sheaves of $\Lambda$-modules on $X$ with morphisms being homotopy classes of morphisms of complexes. Let $\tilde{S}$ be the strict Henselization of $S$ at a geometric point $\bar{s}$ over $s$. Fix notation by the following commutative diagram:

$$
\begin{array}{lllll}
\bar{\eta} & \stackrel{\bar{j}}{\rightarrow} & \tilde{S} & \stackrel{\bar{i}}{\leftarrow} & \bar{s} \\
\downarrow & & \downarrow & & \downarrow \\
\eta & \stackrel{j}{\rightarrow} & S & \stackrel{i}{\leftarrow} & s .
\end{array}
$$

For any complex $K \in \operatorname{ob} C(S, \Lambda)$, we have

$$
\begin{aligned}
R \Gamma\left(\bar{s}, R \Psi_{\eta}(K)\right) & =R \Gamma\left(\bar{s}, \bar{i}^{*} R \bar{j}_{*} \bar{j}^{*}\left(\left.K\right|_{\tilde{S}}\right)\right) \\
& \cong \Gamma\left(\bar{s}, \bar{i}^{*} \bar{j}_{*} \bar{j}^{*}\left(\left.K\right|_{\tilde{S}}\right)\right) \\
& \cong K_{\bar{\eta}} .
\end{aligned}
$$

So, $R \Psi_{\eta}(K)$ corresponds to the complex $K_{\bar{\eta}}$ of $\Lambda$-modules with $\operatorname{Gal}(\bar{\eta} / \eta)$-action. Recall that $R \Phi_{\eta}(K)$ is the mapping cone of the canonical morphism $\bar{i}^{*}\left(\left.K\right|_{\tilde{S}}\right) \rightarrow R \Psi_{\eta}(K)$.

Let $I$ be the inertia subgroup of $\operatorname{Gal}(\bar{\eta} / \eta)$, that is, the kernel of the canonical epimorphism $\operatorname{Gal}(\bar{\eta} / \eta) \rightarrow \operatorname{Gal}(\bar{s} / s)$. The functor

$$
K \mapsto\left(i^{*} K, j^{*} K, i^{*} K \rightarrow i^{*} j_{*} j^{*} K\right)
$$

defines an equivalence of categories from the category $C(S, \Lambda)$ to the category of triples

$$
\left(F, G, F \rightarrow G^{I}\right)
$$


where $F$ is a complex of $\Lambda$-modules with $\operatorname{Gal}(\bar{s} / s)$-action which can be identified with an object in $C(s, \Lambda), G$ is a complex of $\Lambda$-modules with $\operatorname{Gal}(\bar{\eta} / \eta)$-action which can be identified with an object in $C(\eta, \Lambda)$, and $F \rightarrow G^{I}$ is an equivariant morphism complexes. Given an object $K$ in $C(S, \Lambda)$, consider the triples

$$
K^{\prime}=\left(K_{\bar{s}}, K_{\bar{s}}, K_{\bar{s}} \stackrel{\mathrm{id}}{\rightarrow} K_{\bar{s}}\right), \quad K=\left(K_{\bar{s}}, K_{\bar{\eta}}, K_{\bar{s}} \rightarrow K_{\bar{\eta}}^{I}\right) .
$$

Note that the second object is exactly the triple associated to $K$ and hence is denoted by $K$. The first object corresponds to a complex of constant sheaves on $S$. We have a canonical morphism $K^{\prime} \rightarrow K$. Let $K^{\prime \prime}$ be the mapping cone of $K^{\prime} \rightarrow K$. Then the canonical morphism $j_{!} j^{*} K^{\prime \prime} \rightarrow K^{\prime \prime}$ defines a quasi-isomorphism $j_{!} R \Phi_{\eta}(K) \rightarrow K^{\prime \prime}$. We thus have a distinguished triangle

$$
K^{\prime} \rightarrow K \rightarrow j_{!} R \Phi_{\eta}(K) \rightarrow
$$

in the derived category $D(S, \Lambda)$. It gives rise to a distinguished triangle

$$
\begin{aligned}
R \Psi_{\eta_{\infty^{\prime}}}\left(p_{1}^{*} K^{\prime} \otimes^{L} \overline{\mathscr{L}_{\psi}\left(\pi t^{\prime}\right)}\right) & \rightarrow R \Psi_{\eta_{\infty^{\prime}}}\left(p_{1}^{*} K \otimes^{L} \overline{\mathscr{L}_{\psi}\left(\pi t^{\prime}\right)}\right) \\
& \rightarrow R \Psi_{\eta_{\infty^{\prime}}}\left(p_{1}^{*}\left(j ! R \Phi_{\eta}(K)\right) \otimes^{L} \overline{\mathscr{L}_{\psi}\left(\pi t^{\prime}\right)}\right) \rightarrow,
\end{aligned}
$$

which can be identified with a distinguished triangle

$$
\begin{aligned}
R \Phi_{\eta_{\infty^{\prime}}}\left(p_{1}^{*} K^{\prime} \otimes^{L} \overline{\mathscr{L}_{\psi}\left(\pi t^{\prime}\right)}\right) & \rightarrow R \Phi_{\eta_{\infty^{\prime}}}\left(p_{1}^{*} K \otimes^{L} \overline{\mathscr{L}_{\psi}\left(\pi t^{\prime}\right)}\right) \\
& \rightarrow R \Phi_{\eta_{\infty^{\prime}}}\left(p_{1}^{*}\left(j ! R \Phi_{\eta}(K)\right) \otimes^{L} \overline{\mathscr{L}_{\psi}\left(\pi t^{\prime}\right)}\right) \rightarrow
\end{aligned}
$$

because $\overline{\mathscr{L}_{\psi}\left(\pi t^{\prime}\right)}$ vanishes on $S \times{ }_{k} \bar{\infty}^{\prime}$. Since $K^{\prime}$ corresponds to a complex of constant sheaves on $S$, we have

$$
R \Phi_{\eta_{\infty^{\prime}}}\left(p_{1}^{*} K^{\prime} \otimes^{L} \overline{\mathscr{L}_{\psi}\left(\pi t^{\prime}\right)}\right)=0
$$

by $[12,1.3 .1 .2]$. (Note that $[12,1.3 .1 .2]$ holds also for the torsion coefficients $\Lambda$ as its proof in [11, Appendix 2.4] shows.) We thus have

$$
R \Phi_{\eta_{\infty^{\prime}}}\left(p_{1}^{*} K \otimes^{L} \overline{\mathscr{L}_{\psi}\left(\pi t^{\prime}\right)}\right) \cong R \Phi_{\eta_{\infty^{\prime}}}\left(p_{1}^{*}\left(j_{!} R \Phi_{\eta}(K)\right) \otimes^{L} \overline{\mathscr{L}_{\psi}\left(\pi t^{\prime}\right)}\right) .
$$

Lemma 4.2. Let $f: X \rightarrow S$ a morphism, $x$ a $k$-rational point in the special fiber $f^{-1}(s), X_{(x)}$ the Henselization of $X$ at $x, f_{(x)}: X_{(x)} \rightarrow S$ the morphism induced by $f$, and $K \in$ ob $D_{c}^{b}\left(X, \overline{\mathbb{Q}}_{\ell}\right)$. Then we have a canonical isomorphism

$$
R \Phi\left(R f_{(x) *}\left(\left.K\right|_{X_{(x)}}\right)\right) \cong(R \Phi(K))_{x}
$$

Proof. Let $X_{(\bar{x})}$ (resp. $\tilde{S}$ ) be the strict Henselization of $X$ (resp. $S$ ) at a geometric point $\bar{x}$ (resp. $\bar{s}$ ) over $x$ (resp. $s$ ). Since $x$ is a $k$-rational point, we have $X_{(\bar{x})} \cong$ $X_{(x)} \times_{S} \tilde{S}$. Let $f_{(\bar{x})}: X_{(\bar{x})} \rightarrow \tilde{S}$ be the morphism induced by $f$. It can be identified with the base change of $f_{(x)}: X_{(x)} \rightarrow S$. Fix notation by the following commutative diagram:

$$
\begin{array}{rllll}
X_{(\bar{x})} \times_{\tilde{S}} \bar{\eta} & \rightarrow & X_{(\bar{x})} & \leftarrow & X_{(\bar{x})} \times{ }_{\tilde{S}} \bar{s} \\
f_{(\bar{x}), \bar{\eta}} \downarrow & & \downarrow f_{(\bar{x})} & & \downarrow f_{(\bar{x}), \bar{s}} \\
\bar{\eta} & \stackrel{\bar{j}}{\rightarrow} & \tilde{S} & \stackrel{\bar{i}}{\leftarrow} & \bar{s} .
\end{array}
$$


For convenience, denote the restrictions of $K$ to $X_{(x)}, X_{(\bar{x})}$ and $X_{(\bar{x})} \times \tilde{s} \bar{\eta}$ also by $K$. Then $R \Phi\left(R f_{(x) *} K\right)$ is the mapping cone of the canonical morphism

$$
\bar{i}^{*} R f_{(\bar{x}) *} K \rightarrow \bar{i}^{*} R \bar{j}_{*} \bar{j}^{*} R f_{(\bar{x}) *} K .
$$

We have

$$
\begin{aligned}
R \Gamma\left(\bar{s}, \bar{i}^{*} R f_{(\bar{x}) *} K\right) & \cong R \Gamma\left(\tilde{S}, R f_{(\bar{x}) *} K\right) \\
& \cong R \Gamma\left(X_{(\bar{x})}, K\right), \\
R \Gamma\left(\bar{s}, \bar{i}^{*} R \bar{j}_{*} \bar{j}^{*} R f_{(\bar{x}) *} K\right) & \cong R \Gamma\left(\tilde{S}, R \bar{j}_{*} \bar{j}^{*} R f_{(\bar{x}) *} K\right) \\
& \cong R \Gamma\left(\bar{\eta}, \bar{j}^{*} R f_{(\bar{x}) *} K\right) \\
& \cong R \Gamma\left(\bar{\eta}, R f_{(\bar{x}), \bar{\eta} *} K\right) \\
& \cong R \Gamma\left(X_{(\bar{x})} \times_{\tilde{S}} \bar{\eta}, K\right) .
\end{aligned}
$$

It follows that $R \Phi\left(R f_{(x) *} K\right)$ can be identified with the mapping cone of the canonical morphism

$$
R \Gamma\left(X_{(\bar{x})}, K\right) \rightarrow R \Gamma\left(X_{(\bar{x})} \times_{\tilde{S}} \bar{\eta}, K\right) .
$$

The later is exactly $(R \Phi(K))_{x}$.

Under the assumption of Lemma 3.1, let $Y_{(y)}$ be the Henselization of $Y$ at $y$, and let $g_{(y)}: Y_{(y)} \rightarrow S$ be the morphism induced by $g$. Fix notation by the following commutative diagram:

$$
\begin{array}{ccccc}
Y_{(y)} \times_{k} \mathbb{P}_{\left(\infty^{\prime}\right)}^{1} & \stackrel{g_{(y)} \times \mathrm{id}_{\mathbb{P}^{1}}\left(\infty^{\prime}\right)}{\rightarrow} & S \times_{k} \mathbb{P}_{\left(\infty^{\prime}\right)}^{1} & \stackrel{p_{2}}{\longrightarrow} & \mathbb{P}_{\left(\infty^{\prime}\right)}^{1} \\
\operatorname{pr}_{1(y)} \downarrow & & p_{1} \downarrow & & \downarrow \\
Y_{(y)} & \stackrel{g_{(y)}}{\longrightarrow} & S & \rightarrow & \text { Spec } k .
\end{array}
$$

Denote the restriction of $K$ to $Y_{(y)}$ also by $K$. We have canonical morphisms

$$
\begin{aligned}
R \Psi_{\eta_{\infty^{\prime}}} & \left(p_{1}^{*} R g_{(y) *} K \otimes^{L} \overline{\mathscr{L}_{\psi}\left(\pi t^{\prime}\right)}\right) \\
\cong & R \Psi_{\eta_{\infty^{\prime}}}\left(R\left(g_{(y)} \times \operatorname{id}_{\mathbb{P}_{\left(\infty^{\prime}\right)}^{1}}\right) * \operatorname{pr}_{1(y)}^{*} K \otimes^{L} \overline{\mathscr{L}_{\psi}\left(\pi t^{\prime}\right)}\right) \\
& \quad(\text { smooth base change theorem }) \\
\cong & R \Psi_{\eta_{\infty^{\prime}}}\left(R\left(g_{(y)} \times \operatorname{id}_{\mathbb{P}_{\left(\infty^{\prime}\right)}^{1}}\right)_{*}\left(\operatorname{pr}_{1(y)}^{*} K \otimes^{L}\left(g_{(y)} \times \operatorname{id}_{\mathbb{P}_{\left(\infty^{\prime}\right)}^{1}}\right)^{*} \overline{\mathscr{L}_{\psi}\left(\pi t^{\prime}\right)}\right)\right) \\
& \quad(\text { projection formula) } \\
\rightarrow & R\left(g_{(y)} \times \operatorname{id}_{\left.\bar{\infty}^{\prime}\right)}\right)_{*} R \Psi_{\eta_{\infty^{\prime}}}\left(\operatorname{pr}_{1(y)}^{*} K \otimes^{L}\left(g_{(y)} \times \operatorname{id}_{\mathbb{P}_{\left(\infty^{\prime}\right)}^{1}}\right)^{*} \overline{\mathscr{L}_{\psi}\left(\pi t^{\prime}\right)}\right)
\end{aligned}
$$

Since $p_{1}^{*} R g_{(y) *} K \otimes^{L} \overline{\mathscr{L}_{\psi}\left(\pi t^{\prime}\right)}$ and $\operatorname{pr}_{1(y)}^{*} K \otimes^{L}\left(g_{(y)} \times \operatorname{id}_{\mathbb{P}_{\left(\infty^{\prime}\right)}^{1}}\right)^{*} \overline{\mathscr{L}_{\psi}\left(\pi t^{\prime}\right)}$ vanish on the fibers over $\infty^{\prime}$, their vanishing cycles complex and nearby cycles complex coincide. So the composite of the above canonical morphisms can be identified with a canonical morphism

$$
\begin{aligned}
& R \Phi_{\eta_{\infty^{\prime}}}\left(p_{1}^{*} R g_{(y) *} K \otimes^{L} \overline{\mathscr{L}_{\psi}\left(\pi t^{\prime}\right)}\right) \\
& \quad \rightarrow R\left(g_{(y)} \times \operatorname{id}_{\bar{\infty}^{\prime}}\right)_{*} R \Phi_{\eta_{\infty^{\prime}}}\left(\operatorname{pr}_{1(y)}^{*} K \otimes^{L}\left(g_{(y)} \times \operatorname{id}_{\mathbb{P}_{\left(\infty^{\prime}\right)}^{1}}\right)^{*} \overline{\mathscr{L}_{\psi}\left(\pi t^{\prime}\right)}\right) .
\end{aligned}
$$


Applying Lemma 4.1 to the complex $R g_{(y) *} K$ on $S$, we get a canonical isomorphism

$$
R \Phi_{\eta_{\infty^{\prime}}}\left(p_{1}^{*} R g_{(y) *} K \otimes^{L} \overline{\mathscr{L}_{\psi}\left(\pi t^{\prime}\right)}\right) \cong R \Phi_{\eta_{\infty^{\prime}}}\left(p_{1}^{*}\left(j ! R \Phi\left(R g_{(y) *} K\right)\right) \otimes^{L} \overline{\mathscr{L}_{\psi}\left(\pi t^{\prime}\right)}\right) .
$$

By Lemma 4.2, we have

$$
R \Phi\left(R g_{(y) *} K\right) \cong\left(R \Phi_{g}(K)\right)_{y} .
$$

We thus get a canonical morphism

$$
\begin{aligned}
& R \Phi_{\eta_{\infty^{\prime}}}\left(p_{1}^{*}\left(j_{!}\left(R \Phi_{g}(K)\right)_{y}\right) \otimes^{L} \overline{\mathscr{L}_{\psi}\left(\pi t^{\prime}\right)}\right) \\
& \quad \rightarrow R\left(g_{(y)} \times \operatorname{id}_{\bar{\infty}^{\prime}}\right)_{*} R \Phi_{\eta_{\infty^{\prime}}}\left(\operatorname{pr}_{1(y)}^{*} K \otimes^{L}\left(g_{(y)} \times \operatorname{id}_{\mathbb{P}_{\left(\infty^{\prime}\right)}^{1}}\right)^{*} \overline{\mathscr{L}_{\psi}\left(\pi t^{\prime}\right)}\right) .
\end{aligned}
$$

By Lemma 3.1(i) that we have shown at the beginning of this section, this gives rise to a canonical morphism

$$
\begin{aligned}
& R \Phi_{\eta_{\infty^{\prime}}}\left(p_{1}^{*}\left(j_{!}\left(R \Phi_{g}(K)\right)_{y}\right) \otimes^{L} \overline{\mathscr{L}_{\psi}\left(\pi t^{\prime}\right)}\right)_{\left(s, \infty^{\prime}\right)} \\
& \quad \rightarrow R \Phi_{\eta_{\infty^{\prime}}}\left(\operatorname{pr}_{1}^{*} K \otimes^{L}\left(g \times \operatorname{id}_{\mathbb{P}_{\left(\infty^{\prime}\right)}^{1}}\right)^{*} \overline{\mathscr{L}}_{\psi}\left(\pi t^{\prime}\right)\right)_{(y, \infty)} .
\end{aligned}
$$

Under the assumption of Lemma 3.1(iii), taking the $n$th cohomology on both sides and using the definition of $\mathscr{F}^{\left(0, \infty^{\prime}\right)}$ in $[12,2.4 .2 .3]$, we get a canonical morphism

$$
\mathscr{F}^{\left(0, \infty^{\prime}\right)}\left(R^{n-1} \Phi_{g}(K)_{y}\right) \rightarrow R^{n} \Phi_{\eta_{\infty^{\prime}}}\left(\operatorname{pr}_{1}^{*} K \otimes^{L}\left(g \times \operatorname{id}_{\mathbb{P}_{\left(\infty^{\prime}\right)}}\right)^{*} \overline{\mathscr{L}_{\psi}\left(\pi t^{\prime}\right)}\right)_{\left(y, \infty^{\prime}\right)} .
$$

Lemma 4.3. Let $f: X \rightarrow S$ be a proper morphism, and let $K \in \operatorname{ob} D_{c}^{b}\left(X, \overline{\mathbb{Q}}_{\ell}\right)$. Suppose $X$ is regular pure of dimension $n, f$ is smooth at points in $f^{-1}(s)-A$ for a finite set $A$ of $k$-rational points in $f^{-1}(s)$ and the sheaves $\left.\mathscr{H}^{q}(K)\right|_{X-A}$ are lisse for all $q$. Then for all $x \in A$, the canonical morphisms

$$
\begin{aligned}
& R \Phi_{\eta_{\infty^{\prime}}}\left(p_{1}^{*}\left(j_{!}\left(R \Phi_{f}(K)\right)_{x}\right) \otimes^{L} \overline{\mathscr{L}_{\psi}\left(\pi t^{\prime}\right)}\right)_{\left(s, \infty^{\prime}\right)} \\
& \quad \rightarrow R \Phi_{\eta_{\infty^{\prime}}}\left(\operatorname{pr}_{1}^{*} K \otimes^{L}\left(f \times \operatorname{id}_{\mathbb{P}_{\left(\infty^{\prime}\right)}^{1}}\right)^{*} \overline{\mathscr{L}_{\psi}\left(\pi t^{\prime}\right)}\right)_{(x, \infty)}
\end{aligned}
$$

constructed above are isomorphisms, where $\mathrm{pr}_{1}: X \times_{k} \mathbb{P}_{\left(\infty^{\prime}\right)}^{1} \rightarrow X$ is the projection. Suppose furthermore that $K$ is a lisse sheaf. Then the canonical morphisms

$$
\mathscr{F}^{\left(0, \infty^{\prime}\right)}\left(R^{n-1} \Phi_{f}(K)_{x}\right) \rightarrow R^{n} \Phi_{\eta_{\infty^{\prime}}}\left(\operatorname{pr}_{1}^{*} K \otimes^{L}\left(f \times \operatorname{id}_{\mathbb{P}_{\left(\infty^{\prime}\right)}^{1}}\right)^{*} \overline{\mathscr{L}_{\psi}\left(\pi t^{\prime}\right)}\right)_{\left(x, \infty^{\prime}\right)}
$$

are isomorphisms for all $x \in A$.

Proof. The second statement follows from the first one. To prove the first statement, it suffices to prove the above canonical morphisms induce an isomorphism

$$
\begin{array}{rl}
\bigoplus_{x \in A} & R \Phi_{\eta_{\infty^{\prime}}}\left(p_{1}^{*}\left(j_{!}\left(R \Phi_{f}(K)\right)_{x}\right) \otimes^{L} \overline{\mathscr{L}_{\psi}\left(\pi t^{\prime}\right)}\right)_{\left(s, \infty^{\prime}\right)} \\
& \rightarrow \bigoplus_{x \in A} R \Phi_{\eta_{\infty^{\prime}}}\left(\operatorname{pr}_{1}^{*} K \otimes^{L}\left(f \times \mathrm{id}_{\left.\mathbb{P}_{\left(\infty^{\prime}\right)}\right)}\right)^{*} \overline{\mathscr{L}_{\psi}\left(\pi t^{\prime}\right)}\right)_{(x, \infty)} .
\end{array}
$$


Fix notation by the following commutative diagram:

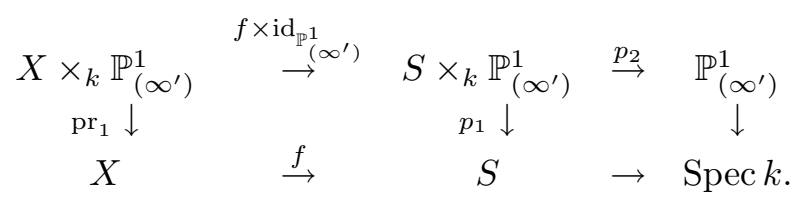

We have canonical isomorphisms

$$
\begin{aligned}
& R \Psi_{\eta_{\infty^{\prime}}}\left(p_{1}^{*} R f_{*} K \otimes^{L} \overline{\mathscr{L}_{\psi}\left(\pi t^{\prime}\right)}\right) \\
& \cong R \Psi_{\eta_{\infty^{\prime}}}\left(R\left(f \times \mathrm{id}_{\mathbb{P}_{\left(\infty^{\prime}\right)}^{1}}\right) * \operatorname{pr}_{1}^{*} K \otimes^{L} \overline{\mathscr{L}_{\psi}\left(\pi t^{\prime}\right)}\right) \quad \text { (smooth base change theorem) } \\
& \cong R \Psi_{\eta_{\infty^{\prime}}}\left(R\left(f \times \operatorname{id}_{\mathbb{P}_{\left(\infty^{\prime}\right)}^{1}}\right) *\left(\operatorname{pr}_{1}^{*} K \otimes^{L}\left(f \times \operatorname{id}_{\mathbb{P}_{\left(\infty^{\prime}\right)}}\right)^{*} \overline{\mathscr{L}_{\psi}\left(\pi t^{\prime}\right)}\right)\right) \\
& \text { (projection formula) } \\
& \cong R\left(f \times \operatorname{id}_{\bar{\infty}^{\prime}}\right)_{*} R \Psi_{\eta_{\infty^{\prime}}}\left(\operatorname{pr}_{1}^{*} K \otimes^{L}\left(f \times \operatorname{id}_{\mathbb{P}_{\left(\infty^{\prime}\right)}^{1}}\right)^{*} \overline{\mathscr{L}_{\psi}\left(\pi t^{\prime}\right)}\right) \\
& \text { (proper base change theorem) }
\end{aligned}
$$

Since $p_{1}^{*} R f_{*} K \otimes^{L} \overline{\mathscr{L}_{\psi}\left(\pi t^{\prime}\right)}$ and $\operatorname{pr}_{1}^{*} K \otimes^{L}\left(f \times \operatorname{id}_{\mathbb{P}_{\left(\infty^{\prime}\right)}^{1}}\right)^{*} \overline{\mathscr{L}_{\psi}\left(\pi t^{\prime}\right)}$ vanish on the fibers over $\infty^{\prime}$, their vanishing cycles complex and nearby cycles complex coincide. So the stalk at $\left(s, \infty^{\prime}\right)$ of the composite of the above canonical isomorphisms can be identified with

$$
\begin{aligned}
& R \Phi_{\eta_{\infty^{\prime}}}\left(p_{1}^{*} R f_{*} K \otimes^{L} \overline{\mathscr{L}_{\psi}\left(\pi t^{\prime}\right)}\right)_{\left(s, \infty^{\prime}\right)} \\
& \quad \cong\left(R\left(f \times \mathrm{id}_{\bar{\infty}^{\prime}}\right)_{*} R \Phi_{\eta_{\infty^{\prime}}}\left(\operatorname{pr}_{1}^{*} K \otimes^{L}\left(f \times \operatorname{id}_{\mathbb{P}_{\left(\infty^{\prime}\right)}^{1}}\right)^{*} \overline{\mathscr{L}_{\psi}\left(\pi t^{\prime}\right)}\right)\right)_{\left(s, \infty^{\prime}\right)} .
\end{aligned}
$$

Applying Lemma 4.1 to the complex $R f_{*} K$ on $S$, we get

$$
R \Phi_{\eta_{\infty^{\prime}}}\left(p_{1}^{*} R f_{*} K \otimes^{L} \overline{\mathscr{L}_{\psi}\left(\pi t^{\prime}\right)}\right) \cong R \Phi_{\eta_{\infty^{\prime}}}\left(p_{1}^{*} j_{!} R \Phi\left(R f_{*} K\right) \otimes^{L} \overline{\mathscr{L}_{\psi}\left(\pi t^{\prime}\right)}\right) .
$$

So we get a canonical isomorphism

$$
\begin{aligned}
& R \Phi_{\eta_{\infty^{\prime}}}\left(p_{1}^{*} j_{!} R \Phi\left(R f_{*} K\right) \otimes^{L} \overline{\mathscr{L}_{\psi}\left(\pi t^{\prime}\right)}\right)_{\left(s, \infty^{\prime}\right)} \\
& \quad \cong\left(R\left(f \times \mathrm{id}_{\bar{\infty}^{\prime}}\right)_{*} R \Phi_{\eta_{\infty^{\prime}}}\left(\operatorname{pr}_{1}^{*} K \otimes^{L}\left(f \times \operatorname{id}_{\mathbb{P}_{\left(\infty^{\prime}\right)}^{1}}\right)^{*} \overline{\mathscr{L}_{\psi}\left(\pi t^{\prime}\right)}\right)\right)_{\left(s, \infty^{\prime}\right)} .
\end{aligned}
$$

By the proper base change theorem and lemma 2.1, we have

$$
\begin{aligned}
R \Phi\left(R f_{*} K\right) & \cong R f_{s *}\left(R \Phi_{f}(K)\right) \\
& \cong \bigoplus_{x \in A}\left(R \Phi_{f}(K)\right)_{x} .
\end{aligned}
$$

By Lemma 3.1(i) that we have shown at the beginning of this section, we have

$$
\begin{aligned}
& \left(R\left(f \times \operatorname{id}_{\bar{\infty}^{\prime}}\right)_{*} R \Phi_{\eta_{\infty^{\prime}}}\left(\operatorname{pr}_{1}^{*} K \otimes^{L}\left(f \times \operatorname{id}_{\mathbb{P}_{\left(\infty^{\prime}\right)}^{1}}\right)^{*} \overline{\mathscr{L}_{\psi}\left(\pi t^{\prime}\right)}\right)\right)_{\left(s, \infty^{\prime}\right)} \\
& \cong \bigoplus_{x \in A} R \Phi_{\eta_{\infty^{\prime}}}\left(\operatorname{pr}_{1}^{*} K \otimes^{L}\left(f \times \operatorname{id}_{\mathbb{P}_{\left(\infty^{\prime}\right)}^{1}}\right)^{*} \overline{\mathscr{L}_{\psi}\left(\pi t^{\prime}\right)}\right)_{\left(x, \infty^{\prime}\right)} .
\end{aligned}
$$


So we can write the isomorphism (4.2) as

$$
\begin{aligned}
& \bigoplus_{x \in A} R \Phi_{\eta_{\infty^{\prime}}}\left(p_{1}^{*} j_{!}\left(R \Phi_{f}(K)\right)_{x} \otimes^{L} \overline{\mathscr{L}_{\psi}\left(\pi t^{\prime}\right)}\right)_{\left(s, \infty^{\prime}\right)} \\
& \cong \bigoplus_{x \in A} R \Phi_{\eta_{\infty^{\prime}}}\left(\operatorname { p r } _ { 1 } ^ { * } K \otimes ^ { L } \left(f \times \mathrm{id}_{\left.\left.\mathbb{P}_{\left(\infty^{\prime}\right)}^{1}\right)^{*} \overline{\mathscr{L}_{\psi}\left(\pi t^{\prime}\right)}\right)_{\left(x, \infty^{\prime}\right)} .}\right.\right.
\end{aligned}
$$

This last isomorphism coincides with the morphism induced by the canonical morphisms (4.1)

$$
\begin{aligned}
& R \Phi_{\eta_{\infty^{\prime}}}\left(p_{1}^{*} j_{!}\left(R \Phi_{f}(K)\right)_{x} \otimes^{L} \overline{\mathscr{L}_{\psi}\left(\pi t^{\prime}\right)}\right)_{\left(s, \infty^{\prime}\right)} \\
& \quad \rightarrow R \Phi_{\eta_{\infty^{\prime}}}\left(\operatorname{pr}_{1}^{*} K \otimes^{L}\left(f \times \mathrm{id}_{\mathbb{P}_{\left(\infty^{\prime}\right)}^{1}}\right)^{*} \overline{\mathscr{L}_{\psi}\left(\pi t^{\prime}\right)}\right)_{\left(x, \infty^{\prime}\right)} .
\end{aligned}
$$

It follows that these canonical morphisms are isomorphisms.

We are now ready to prove Lemma 3.1(ii) and (iv).

Proof of Lemma 3.1(ii) and (iv). The canonical morphism (4.1) defined before Lemma 4.3

$$
\begin{aligned}
& R \Phi_{\eta_{\infty^{\prime}}}\left(p_{1}^{*}\left(j_{!}\left(R \Phi_{g}(K)\right)_{y}\right) \otimes^{L} \overline{\mathscr{L}_{\psi}\left(\pi t^{\prime}\right)}\right)_{\left(s, \infty^{\prime}\right)} \\
& \quad \rightarrow R \Phi_{\eta_{\infty^{\prime}}}\left(\operatorname{pr}_{1}^{*} K \otimes^{L}\left(g \times \operatorname{id}_{\mathbb{P}_{\left(\infty^{\prime}\right)}^{1}}\right)^{*} \overline{\mathscr{L}_{\psi}\left(\pi t^{\prime}\right)}\right)_{(y, \infty)}
\end{aligned}
$$

commutes with the base change on the trait $S$. By [3, Th. finitude 3.7], to prove this canonical morphism is an isomorphism, we can reduce to the case where $k$ is algebraically closed and $S=\operatorname{Spec} k[[\pi]]$. By [5, XVI 2.5], there exists a smooth projective variety $Z$ over $k$, a morphism $h: Z \rightarrow \mathbb{P}_{k}^{1}$ smooth outside a finite set of closed points in $Z$, and a closed point $z$ of $Z$ such that $h(z)=0$ and that there exists an $S$-isomorphism between the Henselization of $Y$ at $y$, and the Henselization of $Z \times_{\mathbb{P}_{k}^{1}} S$ at $z$, where $S$ is regarded as a $\mathbb{P}_{k}^{1}$-scheme by identifying $S$ with the completion of $\mathbb{P}_{k}^{1}$ at 0 . To prove the canonical morphism (3) is an isomorphism is a problem local with respect to the etale topology. So we may reduce to the case where $Y$ is an open subscheme of $X=Z \times_{\mathbb{P}_{k}^{1}} S, y=z$, and $g: Y \rightarrow S$ is induced by the projection $f: X=Z \times_{\mathbb{P}_{k}^{1}} S \rightarrow S$. The morphism $f: X \rightarrow S$ is projective, and is smooth at points in $f^{-1}(s)-A$ for a finite set $A$ of closed points in $f^{-1}(s)$. Our assertion then follows from Lemma 4.3.

\section{Questions and conjectures}

Deligne makes the following conjecture ([2]):

Conjecture 5.1. Let $\Lambda$ be a Noetherian torsion ring with the property that $m \Lambda=0$ for some integer $m$ relatively prime to $p$. let $S_{1}, S_{2}, S$ be Henselian traits over Spec $k$ of equal characteristic $p$, all with residue field $k$, let $s_{1}, s_{2}, s$ be their closed points, and let $a: S_{1} \times_{k} S_{2} \rightarrow S$ be a k-morphism such that $a\left(s_{1}, s_{2}\right)=s$ and such that $a\left(\cdot, s_{2}\right): S_{1} \rightarrow S$ and $a\left(s_{1}, \cdot\right): S_{2} \rightarrow S$ are isomorphisms. For each $i$, let $f_{i}: X_{i} \rightarrow S_{i}$ be a morphism of finite type, let $x_{i}$ be a $k$-rational point in the special fiber such that $\left.f_{i}\right|_{X_{i}-x_{i}}$ is smooth, and let $K_{i} \in$ ob $D_{\text {ctf }}^{b}\left(X_{i}, \mathbb{Z} / \ell^{m}\right)$ such that $\left.\mathscr{H}^{q}\left(K_{i}\right)\right|_{X_{i}-x_{i}}$ are locally constant for all q. (Or more optimistically, one can just assume $K_{i}$ is 
universally locally acylic outside $x_{i}$ relative to $f_{i}$.) Let $\eta_{i}$ be the generic point of $S_{i}$, and let $j_{i}: \eta_{i} \hookrightarrow S_{i}$ be the canonical open immersion.

(i) Under the above conditions, $a \circ\left(f_{1} \times f_{2}\right)$ is locally acyclic relative to $K_{1} \otimes^{L}$ $K_{2}$ outside $\left(x_{1}, x_{2}\right)$, and hence the vanishing cycles complex $R \Phi_{a \circ\left(f_{1} \times f_{2}\right)}\left(K_{1} \otimes^{L} K_{2}\right)$ relative to the morphism $a \circ\left(f_{1} \times f_{2}\right)$ is supported at $\left(x_{1}, x_{2}\right)$. (Confer $[3$, Th. finitude 2.12] for the definition of local acyclicity.)

(ii) There is a canonical isomorphism

$$
\begin{aligned}
& R \Phi_{a}\left(j_{1 !}\left(\left(R \Phi_{f_{1}}\left(K_{1}\right)\right)_{x_{1}}\right) \otimes^{L} j_{2 !}\left(\left(R \Phi_{f_{2}}\left(K_{2}\right)\right)_{x_{2}}\right)\right)_{\left(s_{1}, s_{2}\right)} \\
& \quad \cong\left(R \Phi_{a \circ\left(f_{1} \times f_{2}\right)}\left(K_{1} \otimes^{L} K_{2}\right)\right)_{\left(x_{1}, x_{2}\right)},
\end{aligned}
$$

where $\left(R \Phi_{f_{i}}\left(K_{i}\right)\right)_{x_{i}}(i=1,2)$ are complexes of $\Lambda$-modules with $\operatorname{Gal}\left(\bar{\eta}_{i} / \eta_{i}\right)$-action and are regarded as objects in $D_{c t f}^{b}\left(\eta_{i}, \Lambda\right)$.

Due to the use of the Deligne-Fourier transformation, the method used in this paper is not applicable to solve the conjecture unless $a$ is directly related to the addition of the algebraic group $\mathbb{A}_{k}^{1}$. Moreover, our method works only in characteristic $p$. Another problem that is not treated in the paper is how the Thom-Sebastiani theorem behaves with respect to the variation morphism. This problem over $\mathbb{C}$ is treated by Deligne [1] by topological methods.

\section{Acknowledgments}

I would like to thank L. Illusie for informing me of unpublished work of Deligne on the Thom-Sebastiani theorem, and thank P. Deligne who made many suggestions and comments on earlier versions of this paper. I am also grateful to the referee who points out many subtle points that I ignored. My research is supported by the NSFC.

\section{References}

[1] P. Deligne, Letters to A'Campo (dated Nov. 14, 1972) and to Illusie, (dated May 20, 1999).

[2] P. Deligne, Letters to the author, (dated April 13 and May 23, 2011).

[3] P. Deligne, Cohomologie Étale (SGA $4 \frac{1}{2}$ ), Lecture Notes in Math. 569, Springer-Verlag, 1977.

[4] P. Deligne, La conjecture de Weill II, Publ. Math. IHES. 52 (1981), 313-428.

[5] P. Deligne and N. Katz, Groupes de Monodromie en Géométrie Algébrique (SGA 7), Lecture Notes in Math. 340, Springer-Verlag, 1973.

[6] A. Dimca, A. Némethi, Thom-Sebastiani construction and monodromy of polynomials, Proc. Steklov Inst. Math. 238 (2002), 97-114.

[7] A. Gabrielov, Intersection matrices for certain singularities, Functional Anal. Appl. 7 (1973), 182-193.

[8] A. Grothendieck, Étude locale des schémas et des morphismes de schémas (EGA IV), Publ. Math. IHES (1967), 32.

[9] L. Illusie, Autour du théorème de monodromie locale, Périodes p-adiques, Astérisque 223 (1994), $9-57$.

[10] L. Illusie, Perversité et variation, Manuscripta Math. 112 (2003), 271-295.

[11] N. Katz and G. Laumon, Transformation de Fourier et majoration de sommes exponentielles, Publ. Math. IHES 62 (1985), 361-418.

[12] G. Laumon, Transformation de Fourier, constantes d'équations fontionnelles, et conjecture de Weil, Publ. Math. IHES 65 (1987), 131-210.

[13] A. Némethi, Generalized local and global Sebastiani-Thom type theorems, Compositio Math. 80 (1991), 1-14.

[14] M. Saito, Thom-Sebastiani theorem for Hodge modules, Preprint, Kyoto University, 2012. 
[15] M. Sebastiani and R. Thom, Un résultat sur la monodromie, Inventiones Math. 13 (1971), 90-96.

[16] A. Varchenko, Asymptotic Hodge structure in the vanishing cohomology, Math. USSR Izvestija 18 (1982), 469-512.

Chern Institute of Mathematics and LPMC, Nankai University, Tianjin 300071, People's Republic of China

E-mail address: leisfu@gmail.com; leifu@nankai.edu.cn 
